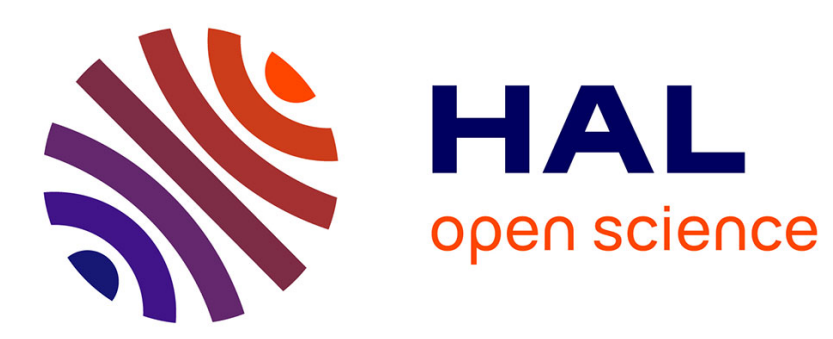

\title{
Asymmetric non-linear forced vibrations of free-edge circular plates. Part 1: Theory
}

Cyril Touzé, Olivier Thomas, Antoine Chaigne

\section{To cite this version:}

Cyril Touzé, Olivier Thomas, Antoine Chaigne. Asymmetric non-linear forced vibrations of freeedge circular plates. Part 1: Theory. Journal of Sound and Vibration, 2002, 258 (4), pp.649-676. 10.1006/jsvi.2002.5143 . hal-00830697

\section{HAL Id: hal-00830697 \\ https://hal-ensta-paris.archives-ouvertes.fr/hal-00830697}

Submitted on 17 Mar 2016

HAL is a multi-disciplinary open access archive for the deposit and dissemination of scientific research documents, whether they are published or not. The documents may come from teaching and research institutions in France or abroad, or from public or private research centers.
L'archive ouverte pluridisciplinaire HAL, est destinée au dépôt et à la diffusion de documents scientifiques de niveau recherche, publiés ou non, émanant des établissements d'enseignement et de recherche français ou étrangers, des laboratoires publics ou privés. 


\title{
ASYMMETRIC NON-LINEAR FORCED VIBRATIONS OF FREE-EDGE CIRCULAR PLATES. PART 1: THEORY
}

\author{
C. Touzé, O. Thomas ${ }^{\dagger}$ And A. Chaigne \\ ENSTA - UME, Chemin de la Hunière, 91761 Palaiseau Cedex, France, and ENST, Département TSI, \\ 46 rue Barrault, 75634 Paris Cedex 13, France. \\ E-mails: touze@ensta.fr, othomas@ensta.fr, chaigne@ensta.fr
}

\begin{abstract}
In this article, a detailed study of the forced asymmetric non-linear vibrations of circular plates with a free edge is presented. The dynamic analogue of the von Kàrmàn equations is used to establish the governing equations. The plate displacement at a given point is expanded on the linear natural modes. The forcing is harmonic, with a frequency close to the natural frequency $\omega_{k n}$ of one asymmetric mode of the plate. Thus, the vibration is governed by the two degenerated modes corresponding to $\omega_{k n}$, which are one-to-one internally resonant. An approximate analytical solution, using the method of multiple scales, is presented. Attention is focused on the case where one configuration which is not directly excited by the load gets energy through non-linear coupling with the other configuration. Slight imperfections of the plate are taken into account. Experimental validations are presented in the second part of this paper.
\end{abstract}

\section{INTRODUCTION}

Circular plates can exhibit large flexural vibrations, of the order of the plate thickness. In this case, a linear model is not sufficient to predict the behaviour of the plate, since typical phenomena such as jump phenomenon, hysteresis or internal resonance are often encountered [1]. In this paper, the dynamic analogue of the von Kàrmàn equations is used to include geometrical non-linearities in the local vibration equations, taking into account the stretching of the mid-plane of the plate.

Attention is focused here on the flexural vibration of circular thin plates, with a free edge, subjected to harmonic excitation. The displacement of a given point of the plate is expanded on the natural modes, which are derived from the linear part of the governing equations. These modes can be either axisymmetric, exhibiting no nodal diameters and hence being independant of the angular co-ordinate $\theta$; or asymmetric, with at least one nodal diameter. For each frequency associated with an asymmetric mode shape there are two corresponding independent modes [2]. Following Tobias and Arnold [3], these two degenerated modes are called the two preferential configurations. If the plate is perfect, the shapes of the two configurations have the same dependence on the radial co-ordinate, and differ only by the position of their nodal diameters, the ones of the first being located on the maxima of amplitude of the second. In the case of a real plate, owing to inevitable small imperfections, it has been shown both theoretically and experimentally that such 
modes have slightly different modal frequencies and that one configuration is moderately rotated with respect to the other, so that the perfect spatial symmetry is broken $[3,4]$.

The axisymmetric vibrations of circular plates have been studied exhaustively in the past; see, for example references [5,6] for one-mode vibration of a plate with various boundary conditions (clamped and simply supported) and without damping, references [7, 8] for multi-mode responses involving internal resonances between axisymmetric modes in the case of a clamped circular plate with model damping, and references [1,9] for general considerations and a survey of the existing literature.

Incidentally, only a few studies have considered the asymmetric case. This is particularly astonishing since for any circular plate, axisymmetric modes are few compared to the number of asymmetric ones. Hence, asymmetric modes are likely to play a preponderant role in the vibrations of plates. The main contribution to the subject is due to Tobias, Arnold and Williams who were the first to consider the non-linear coupling between the two preferential configurations of an asymmetric mode shape [3, 10,11]. They have shown, in particular, that beating travelling waves may appear due to this coupling. This study was conducted on rotating discs clamped at their centre, with a free edge, and the damping was neglected. A recent contribution by Raman and Mote considers the same case of a spinning disc, both theoretically and experimentally $[12,13]$. Dugdale proposed a succinct theoretical work, including damping [14]. Efstathiades brought a major contribution to the subject, since he has taken into account the possible imperfections of the plate in the derivation of the governing equations. He used the von Kàrmàn equations and the Galerkin procedure [4]. Sridhar et al. presented a systematic analysis of the forced response of a clamped circular plate, including damping. They came to the conclusion that a travelling wave component is possible only if an internal resonance exists between modes of frequency well apart from the excitation frequency [15]. Asymmetric linear vibrations of circular plates of linearly varying thickness have also been considered recently in references $[16,17]$. Finally, recent development in computer simulations leads to numerical studies, which generally use a finite element method combined with the harmonic balance method, see for example references [18, 19].

The main purpose of this paper is to present a complete and self-contained analysis of the response of circular thin plates with a free edge and free centre subjected to harmonic excitation, when the excitation frequency is close to one natural frequency. In this paper, only the one-to-one internal resonances between the two preferential configurations of an asymmetric mode are considered. To take into account large deflections, of order of the plate thickness, the non-linear plate model used here is the dynamic analogue of the von Kàrmàn equations [20,21].

This work has been motivated by the fact that only scattered contributions on the subject are available in the literature. Bringing them together, and developing some points that were sometimes overlooked by previous authors allows one to present a detailed analysis in a uniform manner. The solution for the deflection of the plate is expanded into the linear natural modes. The governing partial differential equations lead to a set of second order differential equations, in a similar way to Sridhar et al. reference [15]. The problem is then reduced to the case of a one-mode vibration. The case of an asymmetric mode is studied in detail, with damping and imperfections of the plate taken into account, hence extending the results of Tobias et al., and those presented in references [22, 23]. The temporal part of the deflection is solved by the multiple scales method. A detailed study of the dynamical system arising from this technique is presented, which complements the results of Tobias et al. It is shown, in particular, how the coupling between preferential configurations can give rise to a travelling wave component in the response. An exhaustive 
set of experiments will be described in a companion paper, whose aim is to confirm all the present developments.

\section{PROBLEM FORMULATION}

In what follows, a thin plate of diameter $2 a$ and thickness $h$, made of a homogeneous isotropic material of density $\rho$, Poisson ratio $v$ and Young's modulus $E$, is considered. The only non-zero external loads are normal to the surface of the plate, and represented by a pressure denoted $p(r, \theta, t)$.

\subsection{LOCAL EQUATIONS}

The following non-linear local equations governing the motion of the plate are used:

$$
\begin{gathered}
D \Delta \Delta w+\rho h \ddot{w}=L(w, F)-\mu \dot{w}+p(r, \theta, t), \\
\Delta \Delta F=-\frac{E h}{2} L(w, w),
\end{gathered}
$$

where

$$
L(w, F)=w_{, r r}\left(\frac{F_{, r}}{r}+\frac{F_{, \theta \theta}}{r^{2}}\right)+F_{, r r}\left(\frac{w_{, r}}{r}+\frac{w_{, \theta \theta}}{r^{2}}\right)-2\left(\frac{w_{, r \theta}}{r}-\frac{w_{, \theta}}{r^{2}}\right)\left(\frac{F_{, r \theta}}{r}-\frac{F_{, \theta}}{r^{2}}\right),
$$

$w$ is the vertical displacement at a given point of co-ordinates $(r, \theta)$ of the middle surface of the plate, $D=E h^{3} / 12\left(1-v^{2}\right)$ is the flexural rigidity, $\mu$ is a damping coefficient; $\ddot{w}$ and $w_{, r \theta}$ denote the second partial derivatives with respect to time and spatial co-ordinates, respectively, and

$$
\Delta(\bullet)=(\bullet)_{, r r}+\frac{1}{r}(\bullet)_{, r}+\frac{1}{r^{2}}(\bullet)_{, \theta \theta}
$$

is the Laplacian. The force function $F$ is defined by

$$
N_{r}=\frac{1}{r} F_{, r}+\frac{1}{r^{2}} F_{, \theta \theta}, \quad N_{\theta}=F_{, r r}, \quad N_{r \theta}=N_{\theta r}=\frac{1}{r^{2}} F_{, \theta}-\frac{1}{r} F_{, r \theta},
$$

where $N_{\alpha \beta}, \alpha, \beta \in\{r, \theta\}$ are the membrane forces per unit length in polar co-ordinates, related to the components of the second Piola-Kirchhoff stress tensor $\pi_{\alpha \beta}[24,25]$ :

$$
\left[N_{r}, N_{r \theta}, N_{\theta}\right]=\int_{-h / 2}^{h / 2}\left[\pi_{r}, \pi_{r \theta}, \pi_{\theta}\right] \mathrm{d} z
$$

Equations $(1 \mathrm{a}, \mathrm{b})$ are known as the dynamic analogues of the von-Kàrmàn equations, where damping and forcing have been added. The underlying assumptions are the following. Firstly, the plate is assumed to be thin, i.e., $h / a \ll 1$. Secondly, the KirchhoffLove hypotheses are assumed to be satisfied. Thirdly, only the non-linear terms of lowest order are kept in the expression of the strains as functions of the displacement. Finally, the in-plane and rotatory inertia terms are neglected $[4,15,20,26]$.

Equation (1b) follows from the compatibility equation, and shows the non-linear coupling between the transverse displacement $w$ and the stretching of the mid-plane of the plate, resulting in a non-zero function $F$. 


\subsection{BOUNDARY CONDITIONS}

In the case of a free edge, the external load vanishes at the edge of the plate. The boundary conditions written in cylindrical co-ordinates are, for any point at the edge [26],

$$
\begin{gathered}
N_{r}=N_{r \theta}=0, \\
M_{r}=0, \quad \frac{1}{r} M_{r \theta, \theta}+Q_{r}=0,
\end{gathered}
$$

where $M_{r}$ and $M_{r \theta}$ are the bending and twisting moments and $Q_{r}$ is the transverse shear force. Their definitions in terms of the Piola-Kirchhoff stress tensor are

$$
\left[M_{r}, M_{r \theta}\right]=\int_{-h / 2}^{h / 2}\left[\pi_{r}, \pi_{r \theta}\right] z \mathrm{~d} z, \quad Q_{r}=\int_{-h / 2}^{h / 2} \pi_{z r} \mathrm{~d} z
$$

and they are related to the deflection $w$ by the relations

$$
\begin{gathered}
M_{r}=-D\left(w_{, r r}+\frac{v}{r} w_{, r}+\frac{v}{r^{2}} w_{, \theta \theta}\right), \\
M_{r \theta}=-D(1-v)\left(\frac{w_{, r \theta}}{r}-\frac{w_{, \theta}}{r^{2}}\right) \\
Q_{r}=-D(\Delta w)_{, r} .
\end{gathered}
$$

Combining now equations (6a-d) with equations (4) and (8a, c), one obtains the boundary conditions in terms of $w$ and $F$, for all $\theta$ and $t$ :

$$
\begin{gathered}
F_{, r}+\frac{1}{a} F_{, \theta \theta}=0, \quad F_{, r \theta}-\frac{1}{a} F_{, \theta}=0, \quad \text { at } r=a, \\
w_{, r r}+\frac{v}{a} w_{, r}+\frac{v}{a^{2}} w_{, \theta \theta}=0, \quad \text { at } r=a, \\
w_{, r r r}+\frac{1}{a} w_{, r r}-\frac{1}{a^{2}} w_{, r}+\frac{2-v}{a^{2}} w_{, r \theta \theta}-\frac{3-v}{a^{3}} w_{, \theta \theta}=0, \quad \text { at } r=a .
\end{gathered}
$$

\subsection{DIMENSIONLESS FORM OF THE EQUATIONS}

It is convenient to rewrite the previous equations in terms of dimensionless variables of order $O(1)$. They are denoted by overbars, and defined as follows:

$$
\begin{gathered}
r=a \bar{r}, \quad t=a^{2} \sqrt{\rho h / D} \bar{t}, \quad w=\left(h^{2} / a\right) \bar{w}, \quad F=\left(E h^{5} / a^{2}\right) \bar{F}, \\
\mu=\left[2 E h^{5} / a^{4}\right] \sqrt{\rho h / D} \bar{\mu}, \quad p=\left(E h^{7} / a^{7}\right) \bar{p} .
\end{gathered}
$$

Substituting these variables into equations $(1 \mathrm{a}, \mathrm{b})$ and dropping for simplicity the overbars in the result, we find for all $r, \theta$ and $t$,

$$
\begin{gathered}
\Delta \Delta w+\ddot{w}=\varepsilon[L(w, F)-2 \mu \dot{w}+p], \\
\Delta \Delta F=-\frac{1}{2} L(w, w),
\end{gathered}
$$

where $\varepsilon=12\left(1-v^{2}\right) h^{2} / a^{2}$. The boundary conditions take the same form as in equations (9a-c), with $a=1$. In addition, the solution must be bounded for $r=0$.

One can notice that the von Kàrmàn equations, whose assumptions are summarized in section 2.1, are valid if the magnitude of displacement $w$ is of the order of magnitude of the 
plate thickness $h$. It would then have been apparently logical to choose $\bar{w}=w / h$ as a dimensionless variable for $w$. However, in this case, the parameter $\varepsilon$ would have been equal to $12\left(1-v^{2}\right) a^{2} / h^{2}$, which is large compared to 1 when $h / a \ll 1$, and thus the non-linear terms in equation (12a) would have been of larger order than the linear terms. To derive the analytical perturbation solution of section 6 , the non-linear terms of equation (12a) must be of lower order than the linear terms. If $\bar{w}=w /\left(h^{2} / a\right)$, then $\varepsilon=12\left(1-v^{2}\right) h^{2} / a^{2}$ is small compared to 1 . As already mentioned by Nayfeh et al. [7, 15], the present theory offers corrections to the linear small deflection theory and enables one to predict the typical non-linear phenomena presented in section 6 . This approach is mathematically valid for displacement $w$ of order $h^{2} / a$. In experimental situations, it allows a fair interpretation of observed data up to displacements of the order of the thickness $h$.

\section{MODAL EXPANSION}

The displacement $w$ is written as the product of a function of the space co-ordinates and a function of time. Assuming that the non-linear nature of $w$ is contained in the temporal part of the solution, it is expanded as follows:

$$
w(r, \theta, t)=\sum_{p=1}^{+\infty} \Phi_{p}(r, \theta) q_{p}(t),
$$

where the $q_{p}$ are unknown functions of time and $\Phi_{p}$ are the linear natural modes of the plate with a free edge, corresponding to the eigenfunctions of the linear part of the problem defined by equations (12a) and $(9 b, c)$ (see section A.1).

Substituting equation (13) into equation (12b) leads to

$$
\Delta \Delta F=\sum_{p=1}^{+\infty} \sum_{q=1}^{+\infty} E_{p q}(r, \theta) q_{p}(t) q_{q}(t)
$$

with

$$
E_{p q}=-\frac{1}{2} L\left(\Phi_{p}, \Phi_{q}\right)
$$

Following the same method, an expansion of $F(r, \theta, t)$ is assumed to have the form

$$
F(r, \theta, t)=\sum_{s=1}^{+\infty} \Psi_{s}(r, \theta) \eta_{s}(t)
$$

so that

$$
\Delta \Delta F=\sum_{s=1}^{+\infty}\left[\Delta \Delta \Psi_{s}(r, \theta)\right] \eta_{s}(t),
$$

where the $\eta_{s}$ are unknown functions of time and the $\Psi_{s}$ are chosen so that the following equation is fulfilled, for all $r$ and $\theta$ :

$$
\Delta \Delta \Psi(r, \theta)=\xi^{4} \Psi(r, \theta),
$$

where $\xi$ is an unknown real number. Boundary conditions $(9 \mathrm{a})$, rewritten in terms of $\Psi$ through equation (16), give, for all $\theta$,

$$
\Psi(r=1, \theta)=0, \quad \Psi_{, r}(r=1, \theta)=0 .
$$

It turns out that the $\Psi_{s}$, solutions of equation (18), exhibit the same spatial dependence as the natural modes of a circular plate with clamped edge (section A.2). 
By substituting equation (16) into equation (14), multiplying the result by $\Psi_{b}(b>1)$, integrating on the surface $(\mathscr{S})$ of the plate and using the orthogonality properties of the eigenmodes, one obtains

$$
\eta_{b}(t)=\sum_{p=1}^{+\infty} \sum_{q=1}^{+\infty} G_{p q b} q_{p}(t) q_{q}(t)
$$

with

$$
G_{p q b}=\frac{\iint_{(\mathscr{P})} E_{p q} \Psi_{b} \mathrm{~d} S}{\xi_{b}^{4} \iint_{(\mathscr{S})} \Psi_{u}^{2} \mathrm{~d} S}=-\frac{1}{2} \frac{\iint_{(\mathscr{S})} L\left(\Phi_{p}, \Phi_{q}\right) \Psi_{b} \mathrm{~d} S}{\xi_{b}^{4} \iint_{(\mathscr{P})} \Psi_{u}^{2} \mathrm{~d} S}
$$

so that

$$
\left.F(r, \theta, t)=\sum_{b=1}^{+\infty} \sum_{p=1}^{+\infty} \sum_{q=1}^{+\infty} G_{p g b} q_{p}(t) q_{q}(t)\right) \Psi_{b}(r, \theta)
$$

As is predictable from equation (12b), which shows that $F$ is of order $w^{2}$, one can observe that $F$ is a function of the $q_{p}$ squared only, which corresponds to the order of the non-linear terms. This explains why $F$ and, therefore, the stretching of the mid-plane are neglected in the linear theory.

Substituting equation (22) into equation (12a), multiplying the result by $\Phi_{\alpha}(\alpha>1)$, integrating on the surface $(\mathscr{S})$ of the plate and using the orthogonality properties of the eigenmodes leads to

$$
\ddot{q}_{\alpha}(t)+\omega_{\alpha}^{2} q_{\alpha}(t)=\varepsilon\left[\sum_{p=1}^{+\infty} \sum_{q=1}^{+\infty} \sum_{u=1}^{+\infty} \Gamma_{p q \alpha u} q_{p}(t) q_{q}(t) q_{u}(t)-2 \mu_{\alpha} \dot{q}_{\alpha}(t)+\tilde{Q}_{\alpha}(t)\right]
$$

where $\mu_{\alpha}$ denotes the dimensionless modal damping of the $\alpha$ th mode. The dimensionless pulsation $\omega_{\alpha}$, the coefficients $\Gamma_{p q \alpha u}$ and the forcing $\tilde{Q}_{\alpha}(t)$ are defined as follows:

$$
\begin{gathered}
\omega_{\alpha}^{2}=\frac{\iint_{(\mathscr{S})} \Phi_{\alpha} \Delta \Delta \Phi_{\alpha} \mathrm{d} S}{\iint_{(\mathscr{S})} \Phi_{\alpha}^{2} \mathrm{~d} S}, \quad \tilde{Q}_{\alpha}(t)=\frac{\iint_{(\mathscr{S})} p \Phi_{\alpha} \mathrm{d} S}{\iint_{(\mathscr{S})} \Phi_{\alpha}^{2} \mathrm{~d} S}, \\
\Gamma_{p q \alpha u}=-\frac{1}{2} \sum_{b=1}^{+\infty} \frac{\iint_{(\mathscr{S})} L\left(\Phi_{p}, \Phi_{q}\right) \Psi_{b} \mathrm{~d} S \iint_{(\mathscr{S})} \Phi_{\alpha} L\left(\Phi_{u}, \Psi_{b}\right) \mathrm{d} S}{\xi_{b}^{4} \iint_{(\mathscr{S})} \Phi_{\alpha}^{2} \mathrm{~d} S \iint_{\mathscr{S}} \Psi_{b}^{2} \mathrm{~d} S} .
\end{gathered}
$$

The two partial-differential equations $(12 \mathrm{a}, \mathrm{b})$ have been substituted by the above set of coupled non-linear differential equations (23), whose unknowns are the functions $q_{\alpha}(t)$. One can notice that the mathematical derivations have been kept general. The infinity of modes is a priori supposed to take part in the vibration, the $\alpha$ th differential equation in $q_{\alpha}$ of set (23) governing the contribution of the $\alpha$ th mode.

\section{ONE-MODE VIBRATION}

In this section, the external pressure $p(r, \theta, t)$ is assumed to be equal to zero everywhere, except on a localized surface $S_{d r}$, where the pressure is harmonic with frequency $\Omega$. The area of $S_{d r}$ is assumed to be negligible with respect to the area $\mathscr{S}$ of the plate. The corresponding forcing terms are then $\tilde{Q}_{\alpha}(t)=Q_{\alpha} \cos \Omega t$, where the value of $Q_{\alpha}$ depends on the mode shape $\Phi_{\alpha}$.

The deflection of the plate is governed by the modes which are directly excited by the load near their resonance, and by the modes indirectly excited through an internal 
resonance. The other modes, possibly excited in the transient part of the vibration, decay with time because of the damping. Their magnitudes in the steady state are neglected in the present study. If not, the linear normal modes $\Phi_{p}$ mix with each other in order to give nonlinear modes, whose shapes depend on the vibration amplitude. These effects have been considered both in experimental and theoretical viewpoints, see for example the papers of Shaw and Pierre [27] and references therein, Nayfeh and Nayfeh [28] or Benamar et al. [29]. This approach has not been followed in this article for the two following reasons. First, form an experimental viewpoint, it is shown in reference [29] that the differences between linear and non-linear mode shapes become significant only when the deflection amplitude is larger than the thickness of the plate, which is not the case considered here. Second, as shown in reference [28], corrections due to non-linear modes appear as second order in a perturbation scheme such as the multiple scales method used here. As only a first order multiple scales development is considered in this study, non-linear modes are not taken into account. To summarize, only the modes with natural frequencies close to $\Omega$ are assumed to have significant responses in the steady state. As a consequence, set (23) reduces to the equations governing the response of the excited modes only.

If $\Omega$ is close to the natural frequency $\omega_{0 n}$ of one particular axisymmetric mode, say $\Phi_{0 n}$, set (23) reduces to only one equation. The mode shape of $\Phi_{0 n}$ has no nodal radii, $n$ nodal circles, and depends only on the radial co-ordinate $r$ (Figure 1), so that $\Phi_{0 n}=R_{0 n}(r)$, with $R_{0 n}$ defined in section A.1. Thus, the problem is written as

$$
\begin{gathered}
w(r, t)=R_{0 n}(r) q(t), \\
\ddot{q}(t)+\omega_{0 n}^{2} q(t)=\varepsilon\left[\Gamma q(t)^{3}-2 \mu_{0 n} \dot{q}(t)+Q \cos \Omega t\right] .
\end{gathered}
$$

The mathematical expression of the coefficient $\Gamma$ in equation (26b) is specified in section B.1. Numerical values of $\Gamma$ for different axisymmetric modes are listed in Table 1 . This case of axisymmetric vibration has already been exhaustively studied, and the interested reader can refer to references $[6,7,22]$.

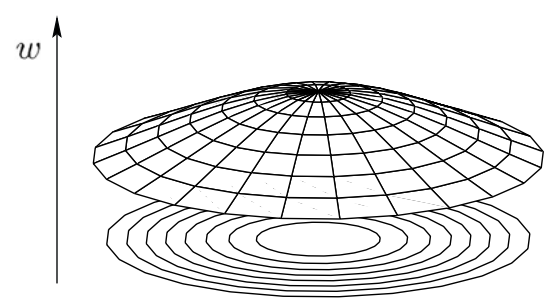

Axisymmetric $\Phi_{01}$ transverse mode

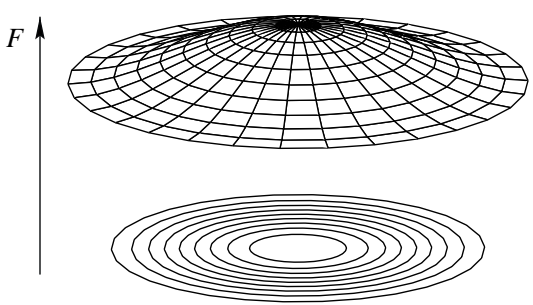

Axisymmetric $\Psi_{01}$ longitudinal mode

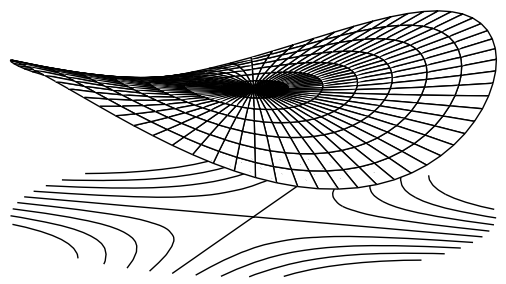

Asymmetric $\Phi_{20}$ transverse mode

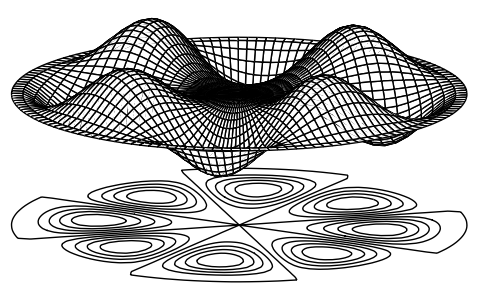

Asymmetric $\Psi_{40}$ longitudinal mode

Figure 1. Mode shapes of typical transverse $(w)$ and longitudinal $(F)$ modes. 
TABLE 1

$\omega_{k n}$ and $\Gamma$ for a few modes. The calculations have been made with $v=0 \cdot 38$

\begin{tabular}{ccccc}
\hline Mode $(k, n)^{\dagger}$ & $\omega_{k n}$ & $\Gamma$ (axisym.) & $\Gamma$ (asym.) & Number of terms ${ }^{\ddagger}$ \\
\hline $2 \cdot 0$ & 5.093 & - & -1.898 & 3 \\
$0 \cdot 1$ & $9 \cdot 175$ & -8.575 & - & 4 \\
$3 \cdot 0$ & 11.90 & - & -17.03 & 4 \\
$1 \cdot 1$ & 20.58 & - & -31.83 & 5 \\
$4 \cdot 0$ & 20.97 & - & -70.72 & 8 \\
$2 \cdot 1$ & 35.21 & -164.4 & -70.01 & 4 \\
$0 \cdot 2$ & 38.61 & -1078.0 & - & 6 \\
$0 \cdot 3$ & 87.92 & & \\
\hline
\end{tabular}

$\dagger k$ : Number of nodal radii; $n$ : number of nodal circles.

${ }^{\ddagger}$ Number of modes $\Psi_{b}$ taken into account in the numerical calculus corresponding to equation (25), to obtain a four significant digit precision.

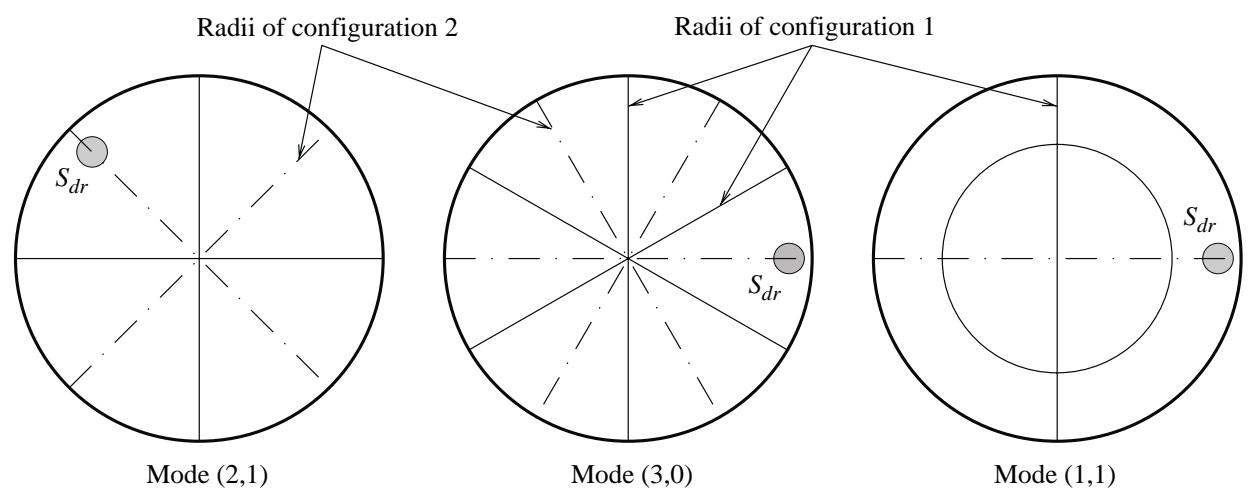

Figure 2. Position of nodal radii of both configurations for various asymmetric modes, in the case of a perfect plate. Position of the surface $S_{d r}$ of the forcing located on a nodal radius of configuration 2.

If $\Omega$ is close to the natural frequency $\omega_{k n}$ of one particular asymmetric mode, the two preferential configurations $\Phi_{1}=\Phi_{k n 1}=R_{k n} \cos k \theta$ and $\Phi_{2}=\Phi_{k n 2}=R_{k n} \sin k \theta$, which are independent and orthogonal, are likely to take part in the vibration. They display $k$ nodal radii, $n$ nodal circles and exhibit the same dependence on $r$. Examples of mode shapes are shown in Figure 1. Expressions of $R_{k n}$ are given in section A.1. Set (23) then reduces to two equations only.

If $S_{d r}$ is assumed to be centred on a nodal radius of configuration $2, \Phi_{2}$, only configuration 1 is directly excited by the external pressure (see Figure 2). Thus, the direct load $Q_{2}$ of configuration $\Phi_{2}$ is zero, and the problem becomes

$$
\begin{gathered}
w(r, \theta, t)=R_{k n}(r)\left[q_{1}(t) \cos k \theta+q_{2}(t) \sin k \theta\right], \\
\ddot{q}_{1}+\omega_{k n}^{2} q_{1}=\varepsilon\left[\Gamma\left(q_{1}^{3}+q_{1} q_{2}^{2}\right)-2 \mu_{k n} \dot{q}_{1}+Q_{1} \cos \Omega t\right], \\
\ddot{q}_{2}+\omega_{k n}^{2} q_{2}=\varepsilon\left[\Gamma\left(q_{2}^{3}+q_{1}^{2} q_{2}\right)-2 \mu_{k n} \dot{q}_{2}\right] .
\end{gathered}
$$

One can notice that the coefficients of the non-linear terms found in equations $(27 \mathrm{~b}, \mathrm{c})$ are equal, and that the coefficients of $q_{2}^{3}$ and $q_{1}^{2} q_{2}$ in equation (27b), and the ones of $q_{1}^{3}$ and $q_{1} q_{2}^{2}$ in equation (27c) are equal to zero. This is a consequence of the particular dependence 
of $\Phi_{1}$ and $\Phi_{2}$ on cosine and sine, and also because the two configurations are situated symmetrically with respect to each other. These results have already been pointed out by Efstathiades [4], and the corresponding mathematical development are given in section B.2. It is probably a general result for all natural modes of a axisymmetric structure, where the dependence in $\theta$ is decoupled from the other spatial co-ordinates. Some values of $\omega_{k n}$ and $\Gamma$ have been computed numerically, and given in Table 1.

Equations $(27 \mathrm{~b}, \mathrm{c})$ show that the deflection of the plate is governed by two modes, $\Phi_{1}$ and $\Phi_{2}$. Because they share similar mode shapes and natural frequencies, one usually says that the plate is subjected to a one-mode vibration.

Equation (22) is convenient for deducing the in-plane vibration of the plate. For this purpose, one has to calculate the values of the coefficients $G_{p q b}$, with $\Phi_{p}$ and $\Phi_{q}$ taken among the two preferential configurations $\Phi_{1}$ and $\Phi_{2}$ with $k$ nodal radii. It can be deduced from section B.2 that the only non-zero $G_{p q b}$ are the ones corresponding to the longitudinal mode $\Psi_{b}$ which are either axisymmetric, or have twice as many nodal radii $(l=2 k)$. It means that the vibration of $w$ on the mode $\Phi_{1}$ (or $\Phi_{2}$ ) leads, via non-linear coupling, to a stretching of the mid-plane based on all its axisymmetric modes $(l=0)$ and all its asymmetric modes of $l=2 k$ nodal radii. Figure 1 shows the transverse mode $\Phi_{20}$ and two associated longitudinal modes $\Psi_{01}$ and $\Psi_{40}$. In addition, it can be derived from equation (22) that the mid-plane is subjected to an oscillatory stretching of pulsation $2 \Omega$, which is two times faster than $w$.

\section{THE CASE OF A SLIGHTLY IMPERFECT PLATE}

In usual experimental conditions, an a priori perfect plate often presents slightly different natural frequencies, and exhibits a small angular shift of the nodal radii compared to the exact symmetric case [3,4]. Thus, in the following mathematical developments, a distinction is made between the two natural frequencies, which will be now denoted by $\omega_{1}$ and $\omega_{2}$, with $\omega_{1} \simeq \omega_{2}$. The angular shifts of the mode shapes of the two configurations are introduced with the help of two angles, $\phi_{1}$ and $\phi_{2}$ (Figure 3), so that

$$
\begin{aligned}
& \Phi_{1}^{*}(r, \theta)=R_{k n}(r) \cos \left(k \theta+\phi_{1}\right), \\
& \Phi_{2}^{*}(r, \theta)=R_{k n}(r) \sin \left(k \theta+\phi_{2}\right) .
\end{aligned}
$$
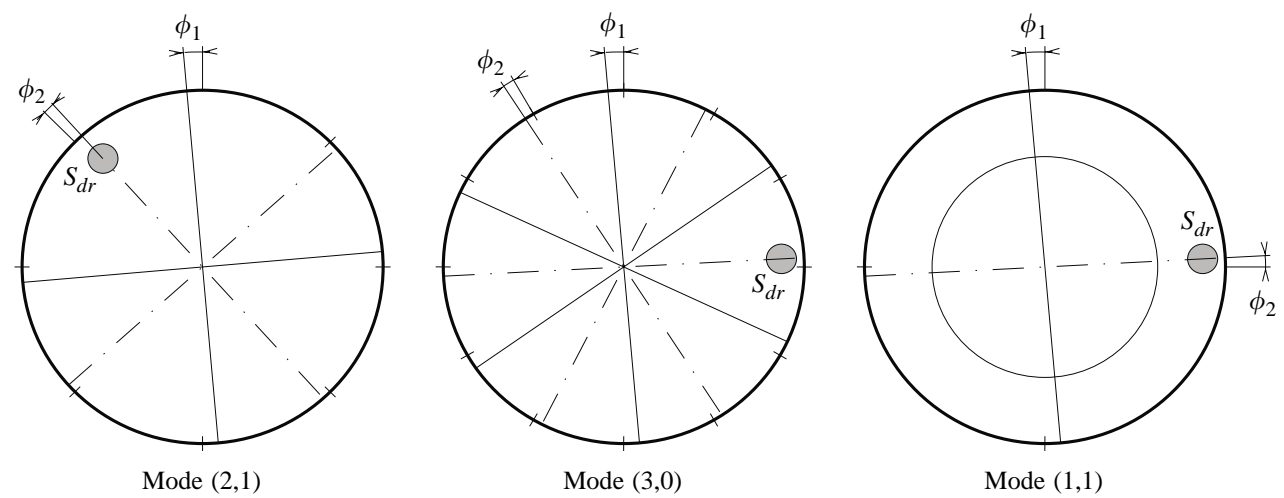

Figure 3. Angular shifts of nodal radii of both configurations for various asymmetric modes, in the case of a real plate. 
The displacement $w$ then becomes

$$
\begin{aligned}
w(r, \theta, t) & =R_{k n}(r)\left[q_{1}^{*}(t) \cos \left(k \theta+\phi_{1}\right)+q_{2}^{*}(t) \sin \left(k \theta+\phi_{2}\right)\right] \\
& =R_{k n}(r)\left[q_{1}(t) \cos (k \theta)+q_{2}(t) \sin (k \theta)\right]
\end{aligned}
$$

with

$$
\left.\left(\begin{array}{l}
q_{1} \\
q_{2}
\end{array}\right)=\begin{array}{cc}
\cos \phi_{1} & \sin \phi_{2} \\
-\sin \phi_{1} & \cos \phi_{2}
\end{array}\right)\left(\begin{array}{l}
q_{1}^{*} \\
q_{2}^{*}
\end{array}\right) .
$$

It is obvious from the above mathematical derivations that set $(27 b, c)$, with two different angular frequencies $\omega_{1}$ and $\omega_{2}$, is sufficient to predict the behaviour of a plate with small imperfections. With $q_{1}$ and $q_{2}$ given by equations $(27 \mathrm{~b}, \mathrm{c})$, one has to invert equation (30) to obtain $q_{1}^{*}$ and $q_{2}^{*}$. These variables are replaced in equation (29a) in order to obtain the displacement $w$. Values of $\omega_{1}, \omega_{2}, \phi_{1}$ and $\phi_{2}$ can be measured on a real plate in experimental conditions.

One can note that the model presented in this paper provides corrections for the perfect plate theory. It is not valid, in particular, if the plate shows large imperfections. This can be the case if holes of significant area compared to the area of the plate are present, or if the boundary conditions are far from being axisymmetric, or if some masses, not negligible compared to the mass of the plate, are added. In those cases, a valid analytical expression of the modes would not be separated in terms of spatial co-ordinates (such as $\Phi(r, \theta)=R(r) f(\theta))$. In particular, the nodal curves would not be perfect circles and radii as in the ideal case.

Finally, in experimental situations, it has been found very difficult to completely cancel the forcing of configuration 2. Therefore, in the following section, a term $Q_{2}$, small compared to $Q_{1}$, will be added in set $(27 \mathrm{~b}, \mathrm{c})$ for the sake of generality.

\section{ANALYTICAL PERTURBATIVE SOLUTION}

This section is devoted to the analysis of the set of coupled Duffing equations derived from equation (27). As this temporal part may occur in many different physical systems once the projection onto the normal modes basis have been carried out, the analysis brought in this section will be provided for variable coefficients. The problem under study is written as

$$
\begin{aligned}
& \ddot{q}_{1}+\omega_{1}^{2} q_{1}=\varepsilon\left[\Gamma_{1} q_{1}^{3}+C_{1} q_{1} q_{2}^{2}-2 \mu \dot{q}_{1}+Q_{1} \cos \Omega t\right], \\
& \ddot{q}_{2}+\omega_{2}^{2} q_{2}=\varepsilon\left[\Gamma_{2} q_{2}^{3}+C_{2} q_{2} q_{1}^{2}-2 \mu \dot{q}_{2}+Q_{2} \cos \Omega t\right],
\end{aligned}
$$

where $\omega_{1} \simeq \omega_{2} \simeq \Omega$.

Most results will be presented for equal coefficients $\left(\Gamma_{1}=\Gamma_{2}=C_{1}=C_{2}\right)$, corresponding to the case of a circular plate with slight imperfections, but some interesting phenomena will be exhibited for other values of the coefficients.

System (31) has already been studied in the past by many different investigators. A study of equation (31) for a subharmonic forced excitation $\left(\Omega \simeq 3 \omega_{1}\right)$, and for the specific case of a perfect circular plate without internal detuning $\left(\omega_{1}=\omega_{2}\right)$, is given in reference [30]. The major contributions have been brought in the study of the vibrations of rectangular plates, where degenerated modes are observed, i.e., two modes having different mode shapes but identical natural frequencies. Yasuda and Asano [31] and Chang et al. [32] performed a thorough analysis of the different behaviours exhibited by a system of form (31). The first authors were primarily interested in the case of an equivalent forcing 
on the two modes $\left(Q_{1} \simeq Q_{2}\right)$. Chang et al. conducted an exhaustive study of the bifurcations. Lewandowski studied in reference [33] the effects of internal resonance on backbone curves with a two harmonics solution. This leads to backbone curves with loops, additional branches and bifurcation points. One-to-one resonances were also studied with parametric excitation, see reference [34] for the case of a nearly square plate with parametric in-plane excitation, or reference [35] for a review of the literature.

The aim of this section is to provide an extension of some of these previously mentioned results. The effect of important physical parameters such as damping, or the internal detuning between the two modal frequencies $\omega_{1}$ and $\omega_{2}$, will be systematically investigated. System (31) will be analytically solved by using the method of multiple scales [1]. Existence conditions for fixed points as well as their stability will be studied. Special emphasis will be put on the loss of stability of single-degree-offreedom solution for the case of a coupled solution. Diagrams will be drawn, which show under which conditions this energy transfer is effective. The case of a softening non-linearity is presented. Experimental validations will be presented in the second part of this paper.

\subsection{MULTIPLE-SCALES SOLUTION}

System (31) is solved by the method of multiple scales [1]. To the first order:

$$
\begin{aligned}
& q_{1}(t)=q_{11}\left(T_{0}, T_{1}\right)+\varepsilon q_{12}\left(T_{0}, T_{1}\right)+O\left(\varepsilon^{2}\right), \\
& q_{2}(t)=q_{21}\left(T_{0}, T_{1}\right)+\varepsilon q_{22}\left(T_{0}, T_{1}\right)+O\left(\varepsilon^{2}\right),
\end{aligned}
$$

where $T_{j}=\varepsilon^{j} t$. Substituting these expressions into equation (31), equating coefficients of like powers of $\varepsilon$ and using $D_{j}=\partial / \partial T_{j}$, one obtains

order $\varepsilon^{0}$ :

$$
\begin{aligned}
& D_{0}^{2} q_{11}+\omega_{1}^{2} q_{11}=0, \\
& D_{0}^{2} q_{21}+\omega_{2}^{2} q_{21}=0,
\end{aligned}
$$

$\operatorname{order} \varepsilon^{1}$ :

$$
\begin{aligned}
& D_{0}^{2} q_{12}+\omega_{1}^{2} q_{12}=-2 D_{0} D_{1} q_{11}+\Gamma_{1} q_{11}^{3}+C_{1} q_{11} q_{21}^{2}-2 \mu D_{0} q_{11}+Q_{1} \cos \Omega t, \\
& D_{0}^{2} q_{22}+\omega_{2}^{2} q_{22}=-2 D_{0} D_{1} q_{21}+\Gamma_{2} q_{21}^{3}+C_{2} q_{21} q_{11}^{2}-2 \mu D_{0} q_{21}+Q_{2} \cos \Omega t .
\end{aligned}
$$

The solutions of equation (33) can be written in the form

$$
\begin{aligned}
& q_{11}(t)=A_{1}\left(T_{1}\right) \exp \left(\mathrm{i} \omega_{1} T_{0}\right)+c . c ., \\
& q_{21}(t)=A_{2}\left(T_{1}\right) \exp \left(\mathrm{i} \omega_{2} T_{0}\right)+c . c .,
\end{aligned}
$$

where $c . c$. stands for complex conjugate. $A_{1}$ and $A_{2}$ are unknown functions of $T_{1}$; their dependence with time will be exhibited when solving the solvability conditions. For this purpose, one introduces the two following detuning parameters:

$$
\omega_{2}=\omega_{1}+\varepsilon \sigma_{1}, \quad \Omega=\omega_{1}+\varepsilon \sigma_{2} .
$$

$\sigma_{1}$ gives a measure of the internal detuning between the two modal configurations. In a completely perfect case, $\omega_{1}=\omega_{2}$, thus $\sigma_{1}=0 . \sigma_{2}$ measures the detuning between the excitation frequency and the natural frequency of configuration (1). Substituting equations (35) into equation (34), and setting all the resonant terms to zero to avoid secular terms in 
the solution gives the solvability conditions

$$
\begin{aligned}
& -2 \mathrm{i} \omega_{1}\left(A_{1}^{\prime}+\mu A_{1}\right)+3 \Gamma_{1} A_{1}^{2} \bar{A}_{1}+C_{1} A_{2}\left(2 A_{1} \bar{A}_{2}+\bar{A}_{1} A_{2} \mathrm{e}^{2 \mathrm{i} \sigma_{1} T_{1}}\right)+\frac{Q_{1}}{2} \mathrm{e}^{\mathrm{i} \sigma_{2} T_{1}}=0, \\
& -2 \mathrm{i} \omega_{2}\left(A_{2}^{\prime}+\mu A_{2}\right)+3 \Gamma_{2} A_{2}^{2} \bar{A}_{2}+C_{2} A_{1}\left(2 A_{2} \bar{A}_{1}+\bar{A}_{2} A_{1} \mathrm{e}^{-2 \mathrm{i} \sigma_{1} T_{1}}\right)+\frac{Q_{2}}{2} \mathrm{e}^{\mathrm{i}\left(\sigma_{2}-\sigma_{1}\right) T_{1}}=0,
\end{aligned}
$$

where ( )' stands now for the derivation with respect to $T_{1}$.

Equations (37) are solved in introducing the polar forms

$$
\begin{aligned}
& A_{1}\left(T_{1}\right)=a_{1}\left(T_{1}\right) \exp \left(\mathrm{i} \theta_{1}\left(T_{1}\right)\right), \\
& A_{2}\left(T_{1}\right)=a_{2}\left(T_{1}\right) \exp \left(\mathrm{i} \theta_{2}\left(T_{1}\right)\right),
\end{aligned}
$$

where the amplitudes $a_{i}$ and the phases $\theta_{i}$ are now real functions of $T_{1}$. Substituting equation (38) into equation (37), identifying real and imaginary parts, one obtains a dynamical system which governs the evolution of amplitudes and phases of the response. This system can be transformed into an autonomous one when defining

$$
\gamma_{1}=\sigma_{2} T_{1}-\theta_{1}, \quad \gamma_{2}=\left(\sigma_{2}-\sigma_{1}\right) T_{1}-\theta_{2} .
$$

One finally obtains

$$
\begin{gathered}
a_{1}^{\prime}=-\mu a_{1}-\frac{C_{1} a_{1} a_{2}^{2}}{2 \omega_{1}} \sin 2\left(\gamma_{2}-\gamma_{1}\right)+\frac{Q_{1}}{4 \omega_{1}} \sin \gamma_{1}, \\
a_{1} \gamma_{1}^{\prime}=\sigma_{2} a_{1}+\frac{3 \Gamma_{1}}{2 \omega_{1}} a_{1}^{3}+\frac{C_{1} a_{1} a_{2}^{2}}{2 \omega_{1}}\left(2+\cos 2\left(\gamma_{2}-\gamma_{1}\right)\right)+\frac{Q_{1}}{4 \omega_{1}} \cos \gamma_{1}, \\
a_{2}^{\prime}=-\mu a_{2}+\frac{C_{2} a_{2} a_{1}^{2}}{2 \omega_{2}} \sin 2\left(\gamma_{2}-\gamma_{1}\right)+\frac{Q_{2}}{4 \omega_{2}} \sin \gamma_{2}, \\
a_{2} \gamma_{2}^{\prime}=\left(\sigma_{2}-\sigma_{1}\right) a_{2}+\frac{3 \Gamma_{2}}{2 \omega_{2}} a_{2}^{3}+\frac{C_{2} a_{2} a_{1}^{2}}{2 \omega_{2}}\left(2+\cos 2\left(\gamma_{2}-\gamma_{1}\right)\right)+\frac{Q_{2}}{4 \omega_{2}} \cos \gamma_{2} .
\end{gathered}
$$

In the more general case, $Q_{1}$ and $Q_{2}$ are not equal to zero. Thus $a_{1}$ and $a_{2}$ are always excited and different from zero. This situation makes it necessary to study the complete four-dimensional system (40) without any simplification, which makes the analytical derivations unsolvable due to the complexity of the involved expressions. The case $Q_{2}=0$ will be studied in detail below. This allows one to simplify some expressions by letting $a_{2}=0$, and to show when the coupling between the two configurations becomes effective. In an experimental context, it is very difficult to ensure that $Q_{2}=0$. The case of a residual excitation on configuration (2) will be taken into account in a companion paper.

Dynamical system (40) exhibits a number of different possible behaviours. For example, limit cycles and chaotic behaviour were found in numerical simulations of equation (40), for specific values of the coefficients $[32,36]$. A complete study of the bifurcation set will not be conducted in this paper. We have been more interested in the situation where an initially non-excited configuration can get energy through non-linear coupling. This phenomenon seems physically more interesting since it shows how energy is transferred between modal configurations. Moreover, all experiments conducted in our laboratory on circular plates, as well as previous experiments carried out on cymbals and gongs [36, 37], showed that an internal resonance is excited before one can observe chaotic behaviour predicted by equations (40). The interested reader can get a meticulous study of the bifurcations exhibited by equations (40) in reference [32]. 


\subsection{FIXED POINTS}

In this section, general fixed-point equations will be established when $Q_{2}=0$. All solution curves will be drawn in the space $\left(\sigma_{2}, a_{1}, a_{2}\right)$, although some figures will show the two projections on the planes $\left(\sigma_{2}, a_{1}\right)$ and $\left(\sigma_{2}, a_{2}\right)$ on the same plot. As long as $a_{2}=0$ (i.e., as the energy transfer has not been realized), only configuration (1) is involved in the vibration: this solution will be referred to as the single-degree-of-freedom (s.d.o.f) solution. When both $a_{1}$ and $a_{2}$ are different from zero, the solution curves will be called the coupled solutions.

General fixed-point equations with $a_{1} \neq 0$ are written as

$$
\begin{gathered}
\mu a_{1}+\frac{C_{1} a_{1} a_{2}^{2}}{2 \omega_{1}} \sin 2\left(\gamma_{2}-\gamma_{1}\right)=\frac{Q_{1}}{4 \omega_{1}} \sin \gamma_{1} \\
\sigma_{2}+\frac{3 \Gamma_{1}}{2 \omega_{1}} a_{1}^{2}+\frac{C_{1} a_{2}^{2}}{2 \omega_{1}}\left(2+\cos 2\left(\gamma_{2}-\gamma_{1}\right)\right)+\frac{Q_{1}}{4 \omega_{1} a_{1}} \cos \gamma_{1}=0 \\
\mu a_{2}=\frac{C_{2} a_{2} a_{1}^{2}}{2 \omega_{2}} \sin 2\left(\gamma_{2}-\gamma_{1}\right), \\
\sigma_{2}-\sigma_{1}+\frac{3 \Gamma_{2}}{2 \omega_{2}} a_{2}^{2}+\frac{C_{2} a_{1}^{2}}{2 \omega_{2}}\left(2+\cos 2\left(\gamma_{2}-\gamma_{1}\right)\right)=0 .
\end{gathered}
$$

Equation (41c) shows explicitly that the possible energy transfer is managed by $C_{2}$. Setting $C_{2}=0$ in equation (41c) shows that the only fixed point for $a_{2}$ is zero. When $C_{2} \neq 0$, a solution with $a_{2} \neq 0$ is possible only if the following equality is fulfilled:

$$
\sin 2\left(\gamma_{2}-\gamma_{1}\right)=\frac{2 \omega_{2} \mu}{C_{2} a_{1}^{2}} .
$$

Equation (42) gives an existence condition for coupled solutions:

$$
a_{1}^{2} \geqslant \frac{2 \omega_{2} \mu}{\left|C_{2}\right|} \text {. }
$$

This condition is only valid for coupled solutions, since in the plane $\left(a_{2}=0\right)$, equation (41c) gives no information.

A second existence condition for general fixed points can be derived from equation (41d). As the absolute value of the cosine function must be less than one, the following condition must be fulfilled:

$$
\left|1+\frac{\omega_{2}}{C_{2} a_{1}^{2}}\left(\sigma_{2}-\sigma_{1}\right)+\frac{3 \Gamma_{2} a_{2}^{2}}{2 C_{2} a_{1}^{2}}\right| \leqslant \frac{1}{2} .
$$

The limiting values of inequality (44) define two paraboloids $\mathscr{P}_{1}$ and $\mathscr{P}_{2}$ in the space $\left(\sigma_{2}, a_{1}, a_{2}\right)$. Branches of solution can develop only inside the region defined by the two paraboloids. Condition (43) shows that coupled solutions can exist only if the amplitude $a_{1}$ is greater than a threshold value. These two conditions define a region $(\mathscr{D})$ where fixed points can exist. This region $(\mathscr{D})$ is represented in Figure 4.

The resonance curve of the s.d.o.f. solution is also shown in Figure 4, where one can see that fixed points for the s.d.o.f. solution can exist outside $(\mathscr{D})$. This is only a calculation artefact due to the use of the polar form (equation (38)). In fact, dynamical system (40) does not accept fixed points outside region $(\mathscr{D})$. However, in the plane $\left(a_{2}=0\right)$, dynamical system (37) accepts the usual fixed points of the s.d.o.f. solution (namely, the resonance curve plotted in Figure 4). This is due to the fact that in polar form, setting $a_{2}=0$ lets $\gamma_{2}$ 




Figure 4. Definition of region $(\mathscr{D})$ in space $\left(\sigma_{2}, a_{1}, a_{2}\right)$ where fixed points of dynamical system $(40)$ can exist. The parameters for this figure have been set to the following values: $\Gamma_{i}=C_{i}=-5, \mu=0 \cdot 15, \omega_{1}=2 \pi, \sigma_{1}=1$. The value of the forcing $Q_{1}$ is set to 10 . The threshold value defined by equation (43) is represented by the horizontal plane.

be undefined. One can find the usual s.d.o.f. solution branches in the plane $\left(a_{2}=0\right)$ by using Cartesian co-ordinates to solve equation (37).

Eliminating the angles in equation (41) gives the general relations for the fixed points in the space $\left(\sigma_{2}, a_{1}, a_{2}\right)$ :

$$
\begin{gathered}
{\left[\sigma_{2}-\sigma_{1}+\frac{3 \Gamma_{2}}{2 \omega_{2}} a_{2}^{2}+\frac{C_{2} a_{1}^{2}}{\omega_{2}}\right]^{2}+\mu^{2}=\frac{C_{2}^{2} a_{1}^{4}}{4 \omega_{2}^{2}},} \\
\frac{Q_{1}^{2}}{16 \omega_{1}^{2}}=\mu^{2} a_{1}^{2}\left[1+\frac{C_{1} \omega_{2} a_{2}^{2}}{C_{2} \omega_{1} a_{1}^{2}}\right]^{2}+\left[\frac{3 \Gamma_{1}}{2 \omega_{1}} a_{1}^{3}-\frac{3 \Gamma_{2} C_{1}}{2 C_{2} \omega_{1}} \frac{a_{2}^{4}}{a_{1}}+\sigma_{2} a_{1}+\left(\sigma_{1}-\sigma_{2}\right) \frac{\omega_{2} C_{1} a_{2}^{2}}{\omega_{1} C_{2} a_{1}}\right]^{2} .
\end{gathered}
$$

Setting $a_{2}=0$ in equation (45b) gives the usual relation for the fixed points of the s.d.o.f. solution. The coupled solutions are solution of equation (45). The stability of the different solution branches will now be investigated. Analytically, we will restrict ourselves to the stability analysis in the plane $\left(a_{2}=0\right)$. General stability of coupled solutions will be determined numerically.

\subsection{STABILITY ANALYSIS}

In this section, we will show that the s.d.o.f. solution loses stability through pitchfork bifurcation. First, the location where coupled solution can start to develop from the plane 


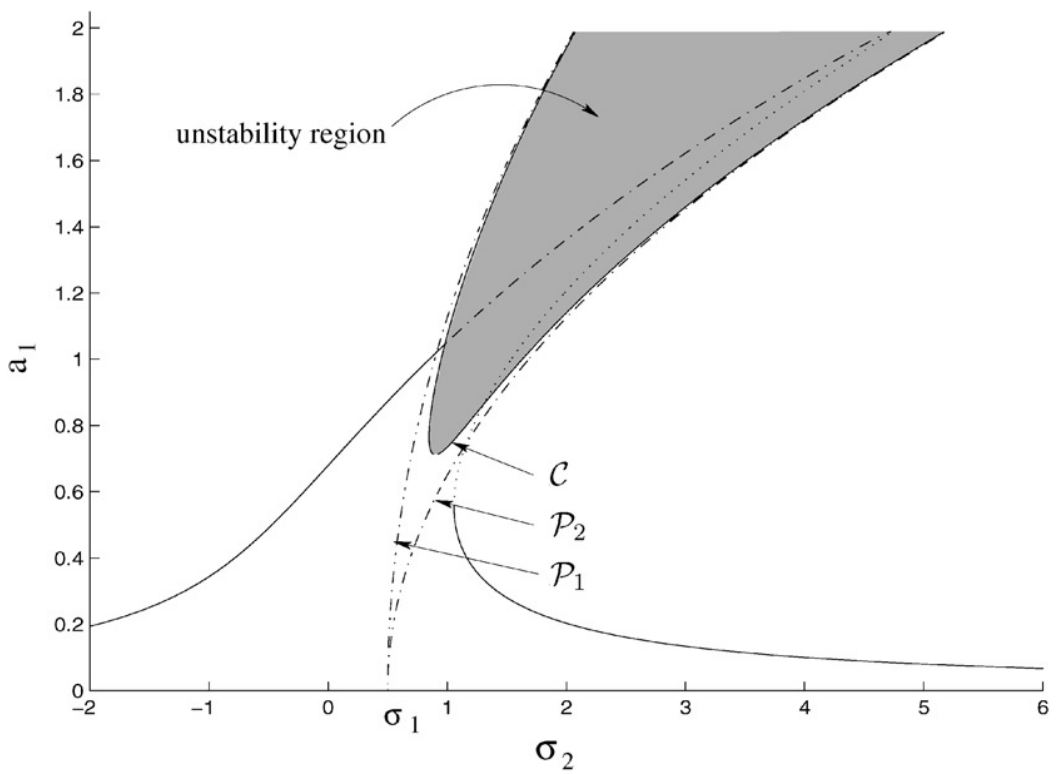

Figure 5. Definition of the unstability region in plane $\left(a_{2}=0\right)$. The dashed lines represent the trace of $\mathscr{P}_{1}$ and $\mathscr{P}_{2}$ on plane $\left(a_{2}=0\right)$. The curve $\mathscr{C}$ is the line where coupled-mode solution can arise. When $\mu=0$, the two regions are identical. The s.d.o.f resonance curve is shown with the following convention: dotted line for unstable state with respect to configuration (1), dash-dotted line for unstable states due to coupling with configuration (2). The following parameters values have been used: $\Gamma_{1}=\Gamma_{2}=C_{1}=C_{2}=-5, \omega_{1}=2 \pi, \mu=0 \cdot 2, \sigma_{1}=0 \cot 5, Q_{1}=10$ and $\varepsilon=0 \cdot 1$.

$\left(a_{2}=0\right)$ are found by setting $a_{2}=0$ in equation (45a). This gives the two branches

$$
\sigma_{2}=\sigma_{1}-\frac{C_{2} a_{1}^{2}}{\omega_{2}} \pm \sqrt{\frac{C_{2}^{2} a_{1}^{4}}{4 \omega_{2}^{2}}-\mu^{2}} .
$$

The curve $\mathscr{C}$, which connects the two branches defined by equation (46), is delimiting a region inside which coupled-mode solutions can arise. We will show next that the s.d.o.f. solution in this region is unstable with respect to perturbation in the $a_{2}$ direction. Hence this domain will be called the unstability region. One can notice that the minimum value of $\mathscr{C}$ is obtained for $a_{1}^{2}=2 \omega_{2} \mu /\left|C_{2}\right|$, which is fully consistent with condition (43). Setting $\mu=0$ in equation (46) gives the two relationships that define the trace of the two paraboloids $\mathscr{P}_{1}$ and $\mathscr{P}_{2}$ in the plane $\left(a_{2}=0\right)$, as can be seen in Figure 5. Hence, the effect of the damping is to make a distinction between region $(\mathscr{D})$ where fixed points for equation (40) exist, and the unstability region. This demonstration will be finalized by conducting a linear stability analysis in the plane $\left(a_{2}=0\right)$.

The Jacobian matrix $\mathscr{J}$ of equation (40) with $a_{2}=0$ is written as

$$
\mathscr{J}=\left(\begin{array}{cccc}
-\mu & -\sigma_{2} a_{1}-\frac{3 \Gamma_{1}}{2 \omega_{1}} a_{1}^{3} & 0 & 0 \\
\frac{\sigma_{2}}{a_{1}}+\frac{9 \Gamma_{1}}{2 \omega_{1}} a_{1} & -\mu & 0 & 0 \\
0 & 0 & -\mu+\frac{C_{2} a_{1}^{2}}{2 \omega_{2}} \sin 2\left(\gamma_{2}-\gamma_{1}\right) & 0 \\
\frac{2\left(\sigma_{1}-\sigma_{2}\right)}{a_{1}} & \frac{C_{2} a_{1}^{2}}{\omega_{2}} \sin 2\left(\gamma_{2}-\gamma_{1}\right) & 0 & -\frac{C_{2} a_{1}^{2}}{\omega_{2}} \sin 2\left(\gamma_{2}-\gamma_{1}\right)
\end{array}\right)
$$


One can see that the upper-left square block (two-dimensional) of the Jacobian matrix is only related to the stability of the s.d.o.f. solution. This part is exactly the same as that calculated for a single Duffing equation, and it governs the stability of the different solution branches for the s.d.o.f. case. The lower-right part of $\mathscr{J}$ governs the stability of the s.d.o.f. solution with respect to disturbances due to the second resonant oscillator. The eigenvalues associated to this block are

$$
\begin{aligned}
& \lambda_{1}=-\mu+\frac{C_{2} a_{1}^{2}}{2 \omega_{2}} \sin 2\left(\gamma_{2}-\gamma_{1}\right), \\
& \lambda_{2}=-\frac{C_{2} a_{1}^{2}}{\omega_{2}} \sin 2\left(\gamma_{2}-\gamma_{1}\right) .
\end{aligned}
$$

One can see that if the oscillators are undamped, the eigenvalues have opposite signs, and thus the s.d.o.f. solution is unstable. In a conservative case, the situation is the following: curve $\mathscr{C}$ is identical to $\mathscr{P}_{1}$ and $\mathscr{P}_{2}$, thus as soon as the s.d.o.f. resonance curve comes into region $(\mathscr{D})$, it becomes unstable with respect to perturbations due to the coupling with the second oscillator. When damping is considered, $\mathscr{C}$ and $\mathscr{P}_{1} \cup \mathscr{P}_{2}$ are not identical anymore. As long as the resonance curve does not cross $\mathscr{C}$, it remains stable, as it can be seen on the eigenvalues, where the occurrence of $\mu$ lets a portion of phase space where the two eigenvalues are still negative. Finally, one can study under which condition one of the eigenvalues vanishes by forming the product $\lambda_{1} \lambda_{2}$. Eliminating the angles in favour of the other parameters and setting $\lambda_{1} \lambda_{2}=0$ yields equation (46). Thus curve $\mathscr{C}$ is not only the place where coupled solutions can arise, but also the location where the s.d.o.f solution loses its stability through pitchfork bifurcation.

\subsection{GENERALIZED STABILITY CURVE}

A generalized stability curve is presented in Figure 6 in the space $\left(\sigma_{2}, a_{1}, a_{2}\right)$. It can be seen that as soon as the s.d.o.f solution crosses the unstability region, the stable solution

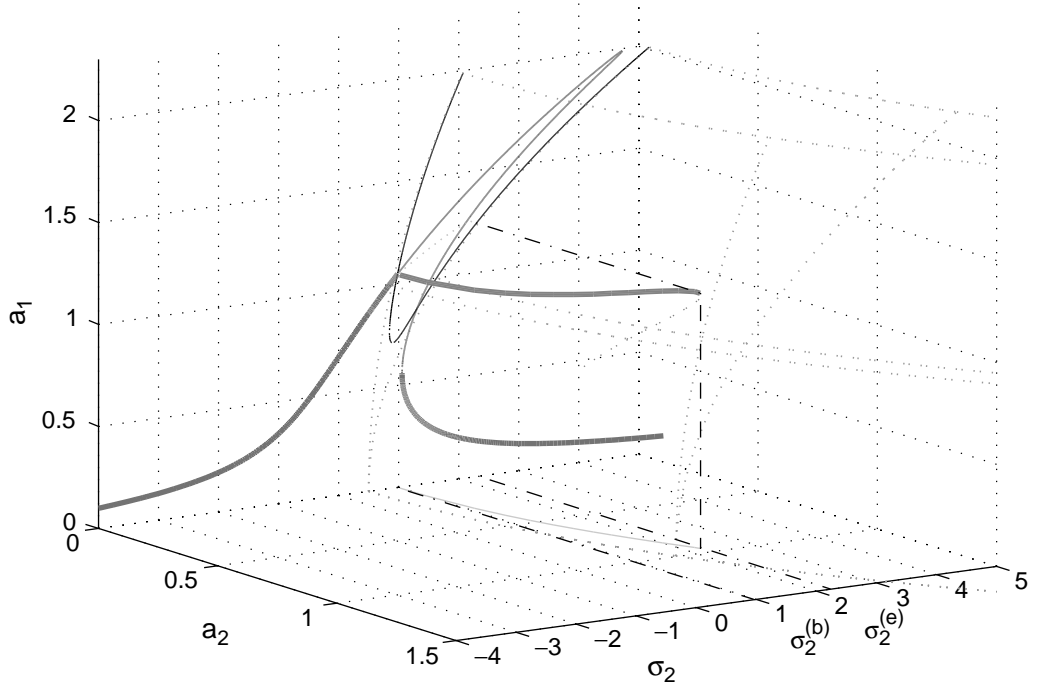

Figure 6. Generalized stability curve for $\Gamma_{1}=\Gamma_{2}=C_{1}=C_{2}=-5, \omega_{1}=2 \pi, \sigma_{1}=0 \cdot 5, \varepsilon=0 \cdot 1, \mu=0 \cdot 2$ and $Q_{1}=10\left(Q_{2}=0\right)$. All the solution branches remain inside region $\mathscr{D}$. Stable solutions are represented with bold lines. The coupled-mode branches develop between $\sigma_{2}^{(b)}$ and $\sigma_{2}^{(e)}$. All the other branches are unstable. 

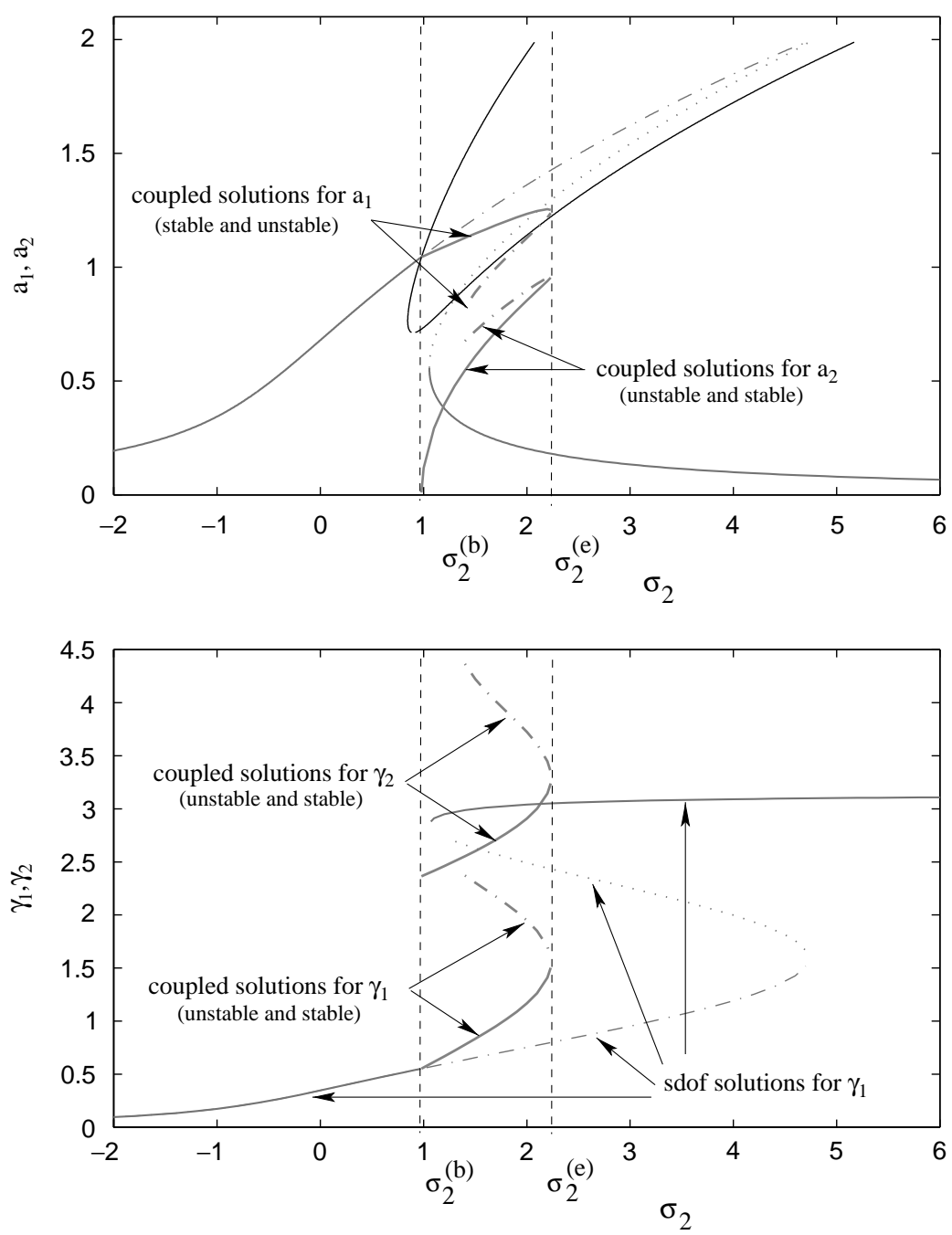

Figure 7. Generalized stability curve for $\Gamma_{1}=\Gamma_{2}=C_{1}=C_{2}=-5, \omega_{1}=2 \pi, \sigma_{1}=0.5, \varepsilon=0.1, \mu=0.2$ and $Q_{1}=10\left(Q_{2}=0\right)$.

becomes the coupled one, which then gets out of the plane $\left(a_{2}=0\right)$. The stability of the two branches of coupled solutions was numerically investigated using the software dstool [38].

The coupled-mode solution arises at $\sigma_{2}^{(b)}$, which is the value of the detuning for the beginning of the unstability region, and disappears at $\sigma_{2}^{(e)}$, a value that cannot be determined analytically.

The projections on the two planes $\left(a_{2}=0\right)$ and $\left(a_{1}=0\right)$ are represented on Figure 7 , where the values of the angles $\gamma_{1}$ and $\gamma_{2}$ have also been reported. The typical behaviour of the phase for the s.d.o.f solution is clearly visible for $\gamma_{1}$. The unstability due to the presence of the second resonant oscillator is shown as the dash-dotted line. Coupled-mode solutions develop between $\sigma_{2}^{(b)}$ and $\sigma_{2}^{(e)}$. They exhibit a phase difference which is nearly equal to $\pi / 2$. This behaviour is expressed in equation (42). When damping is absent, and for the case considered here, the phases of the two configurations are shifted by exactly $\pi / 2$. In fact, when $\mu=0$, coupled-mode solutions develop when the s.d.o.f solution crosses 



Figure 8. Resonance curves with the unstability region (first row) and pitchfork bifurcation curves (solid lines) as well as saddle-node bifurcation curves (dash-dotted lines) in the plane $\left(\sigma_{2}, Q_{1}\right)$, for three different values of the internal detuning. From left to right: $\sigma_{1}=-0 \cdot 5,0.8$ and 2. In the first row, the amplitude of the excitation is fixed at $Q_{1}=10$.

$\mathscr{P}_{1}$. On this paraboloid, we have $\cos 2\left(\gamma_{2}-\gamma_{1}\right)=-1$. According to equation (42), coupled-mode solutions stay on $\mathscr{P}_{1}$ and thus $\gamma_{2}-\gamma_{1}=\pi / 2$. When $\mu \neq 0$, this is not true anymore, and the phases differ from $\pi / 2$ in inverse proportion with $a_{1}^{2}$.

\subsection{EFFECT OF THE INTERNAL DETUNING $\sigma_{1}$, DAMPING $\mu$ AND FORCING $Q_{1}$}

The place where pitchfork and saddle-node bifurcations occur for the s.d.o.f solution can be represented in the parameter space $\left(\sigma_{2}, Q_{1}\right)$. The pitchfork bifurcations are given by the intersection of equation (46) with the s.d.o.f resonance curve defined by

$$
\left.a_{1}^{2} \quad \mu^{2}+\left(\sigma_{2}+\frac{3 \Gamma_{1}}{2 \omega_{1}} a_{1}^{2}\right)^{2}\right)=\frac{Q_{1}^{2}}{16 \omega_{1}^{2}} .
$$

The saddle-node bifurcations (corresponding to the vertical tangent in the s.d.o.f resonance curve) are found by differentiating equation (49) and letting $\partial \sigma_{2} / \partial a_{1}=0$. This gives the relationships

$$
a_{1(S N)}^{2}=\frac{2 \omega_{1}}{9 \Gamma_{1}}\left[-2 \sigma_{2} \pm \sqrt{\sigma_{2}^{2}-3 \mu^{2}}\right],
$$

where $(S N)$ stands for saddle node. These curves are represented in the second row of Figure 8 for different values of the internal detuning $\sigma_{1}$, and for $\Gamma_{1}=-5, C_{2}=-4$, $\omega_{1}=2 \pi$ and $\mu=0 \cdot 15$. The corresponding resonance curves for $Q_{1}=10$ are shown in the first row. From left to right, we have, successively: $\sigma_{1}=-0.5,0.8$ and 2 . One can see that the unstability region depends only on three parameters: the internal detuning $\sigma_{1}$, the coupling coefficient $C_{2}$ and the damping $\mu$. 

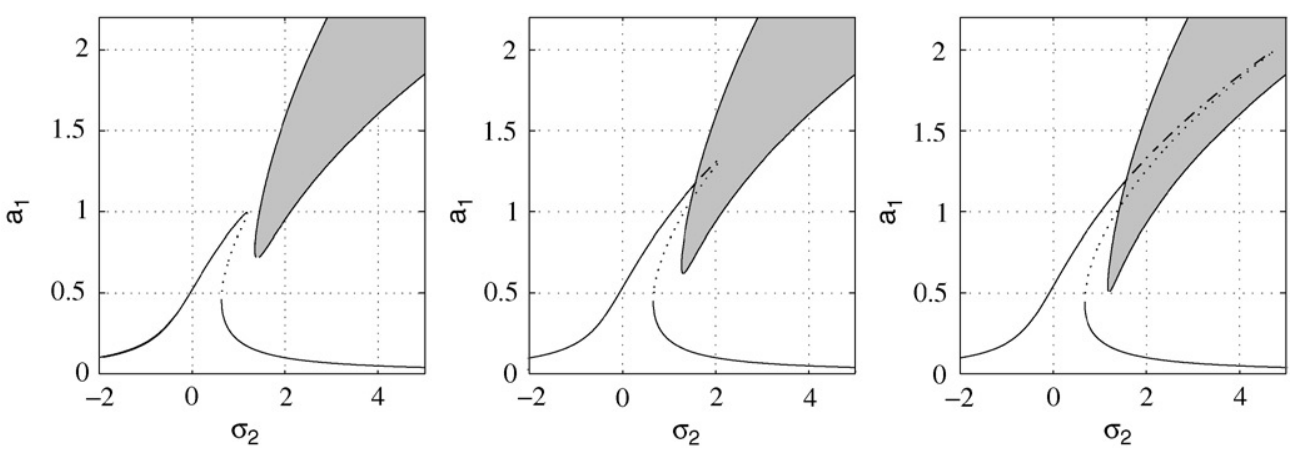

Figure 9. Effect of the damping on both the s.d.o.f resonance curve and the unstability region. $Q_{1}=5$, $\Gamma_{1}=C_{2}=-5, \omega_{1}=2 \pi, \sigma_{1}=1$. From left to right: $\mu=0 \cdot 2,0 \cdot 15$ and $0 \cdot 1$.

The effect of the amplitude of the forcing $Q_{1}$ can be easily seen in Figure 8 . As the unstability region does not depend on $Q_{1}$, the pitchfork bifurcation curves give the limiting value for which the energy transfer from the excited oscillator to the second one is possible. For $\sigma_{1}=-0.5$, for example, a magnitude of $Q_{1}=10$ is not sufficient for obtaining coupled solutions. The critical value is given by the minimum of the pitchfork curve, corresponding to $Q_{1}=12.9$. It should be pointed out that increasing the value of $\left|\sigma_{1}\right|$ leads to consider larger forcing amplitudes to make the coupling effective. This is particularly pronounced when $\sigma_{1}$ is negative in the hardening case, and it will be illustrated in the next paper.

The damping coefficient $\mu$ acts both on the s.d.o.f resonance curve and on the unstability region, which is not the case for $Q_{1}$. Increasing $\mu$ tends to raise the minimum value of the unstability region (this can be seen on condition (43)) and to lower the maximum value of the s.d.o.f resonance curve. Increasing the damping is more dramatic than just decreasing the forcing $Q_{1}$ for coupled-mode solutions to develop (see Figure 9).

\subsection{STUDY OF UNCOMMON CASES}

The aim of this section is to show that dynamical system (40) can exhibit a number of different possible solutions when varying the coefficients. As already mentioned, a complete study of the bifurcations of the coupled solutions will not be conducted here, the reader is referred to reference [32] for global bifurcation sets in the case of rectangular plates. The goal here is to investigate the stability of the s.d.o.f solution. For this purpose, we will examine some generalized stability curves in two different cases.

First, the case of a softening non-linearity will be exhibited. This case can be encountered when studying the rolling motion of a ship (see reference [1] for example), or the non-linear vibrations of circular cylindrical shells [39].

A generalized stability curve is shown in Figure 10 where the damping and the internal detuning have been chosen so that the crossing of the s.d.o.f solution with the unstability region is narrow. This example shows that a narrow intersection is sufficient for a coupled solution to develop, and that a stable s.d.o.f solution and a stable coupled solution can coexist for a wide range of the detuning parameter $\sigma_{2}$. This feature is of course not specific to the softening case. General behaviour reported for the hardening case can be transposed to the softening case. 


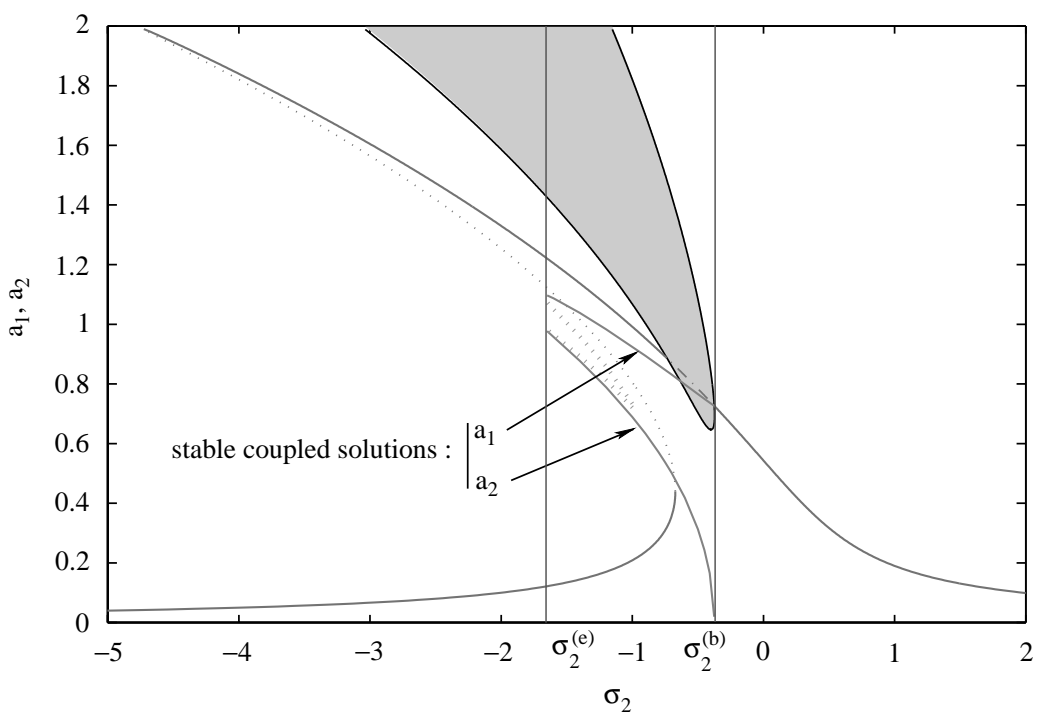

Figure 10. Generalized stability curve in the softening case: $\Gamma_{1}=\Gamma_{2}=5, C_{1}=C_{2}=3, \omega_{1}=2 \pi, \sigma_{1}=-0 \cdot 2$, $\mu=0.1$ and $Q_{1}=5$. For the sake of clarity, only a limited number of unstable coupled solutions have been reported.

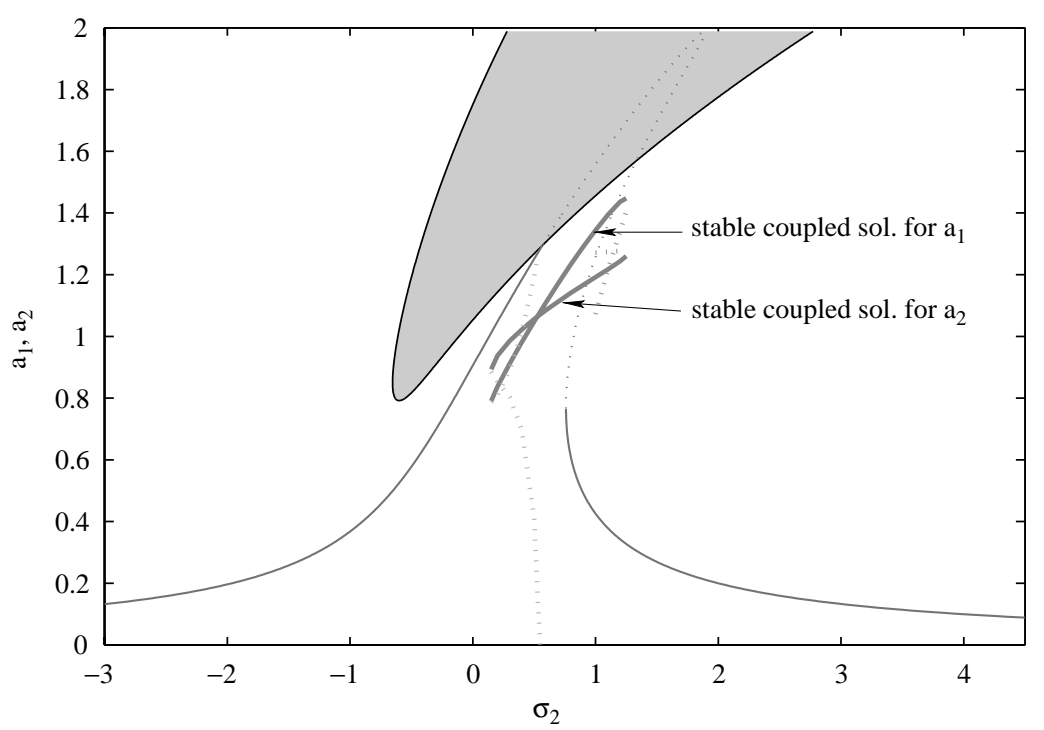

Figure 11. Generalized stability curve for the following values of the parameters: $\Gamma_{1}=C_{1}=-2, \Gamma_{2}=C_{2}=$ $-4, \omega_{1}=2 \pi, \sigma_{1}=-1, \mu=0.2$ and $Q_{1}=10$. Two unstable coupled branches are found, which are represented by dashed lines. The first one arises from the intersection with the unstability region. The second one collapses with the stable coupled solution for $\sigma_{2}=1 \cdot 25$.

Finally, a case where an unusual jump phenomenon can occur is exhibited in Figure 11. As mentioned earlier, the stability of the coupled solutions has not been analytically studied due to the complexity of the algebraic manipulations involved. The particularity of the case exhibited here is the unstability of the coupled solution which arises from the 
intersection of the s.d.o.f resonance curve with the unstability region. The coupled-mode stable solutions are not connected to the plane $\left(a_{2}=0\right)$, as usually seen. This feature has been observed when the s.d.o.f solution crosses the unstability region by the second condition defined by equation (46). In this case, the value of $\cos 2\left(\gamma_{2}-\gamma_{1}\right)$ is near +1 , and not -1 .

A jump phenomenon occurs when increasing the frequency of the forcing at the external border of the unstability region: $a_{1}$ jumps from its s.d.o.f value to the lower stable coupled solution. Meanwhile, $a_{2}$ jumps from zero to its coupled value. This could be experimentally observed when linking together two non-linear cubic oscillators with parameters values that share the same properties as the one exhibited here.

\section{SOLUTION FOR THE DEFLECTION}

Following equations (32), (35), (38) and (27a), to the first approximation, a solution for the steady state deflection $w$ can be written in the form:

$$
w(r, \theta)=R_{k n}(r)\left[2 a_{1} \cos \left(\Omega t-\gamma_{1}\right) \cos k \theta+2 a_{2} \cos \left(\Omega t-\gamma_{2}\right) \sin k \theta\right] .
$$

$w$ results from the superposition of the two preferential configurations, sometimes denoted as two stationary waves. With the help of little algebra, the deflection $w$ can be rewritten in the form

$$
w(r, \theta)=R_{k n}(r)\left[2 a_{3}(\theta) \cos \left(\Omega t-\varepsilon k \theta-\gamma_{3}(\theta)\right)\right],
$$

where $a_{3}$ and $\gamma_{3}$ are constant when $\gamma_{2}-\gamma_{1}=\varepsilon \pi / 2, a_{1}=a_{2}$ and $\varepsilon= \pm 1$. Thus, the deflection can be viewed as a shape $R_{k n}(r) \cos k \theta$ progressing round the disc at speed $\Omega / k$, sometimes called a travelling wave. It travels clockwise or anticlockwise, depending on the sign of $\varepsilon$. If $a_{1} \neq a_{2}$ and $\gamma_{2}-\gamma_{1} \neq \varepsilon \pi / 2, a_{3}$ and $\gamma_{3}$ are slowly varying functions of $k \theta$, and a travelling wave is still obtained, with its amplitude and speed slightly beating around their average value [3]. Section 6 shows that travelling waves can be obtained in the range of the coupled solutions, which has been verified during experiments.

\section{CONCLUSION}

An exhaustive study of the one-mode asymmetric non-linear forced vibrations of freeedge circular plates has been presented in this paper, bringing together scattered contributions on the subject and revisiting a number of points which were overlooked by previous authors.

This research was initiated by the study of non-linear percussion instruments, a family in which one can find cymbals and gongs [36,37]. Non-linear effects are of prime importance for the sound generation in those instruments where the vibration amplitudes are sometimes larger than the thickness. In the context of developing a physical model that would account for the non-linear phenomena exhibited by those instruments, the first step of the modelling procedure (limitation to the one-mode vibration case) is exposed here. As the energy transfer and the selection of modes in multi-mode responses are both governed by eigenfrequency relationships, irrespective of the mode shape (as predicted by normal form theory, for example reference [40], or as observed in experiments [36,37]), it has been necessary to develop first a complete study of the one-mode asymmetric case.

To remain close to the case of percussion instruments, original developments have been performed in this study for the modal expansion with free-edge boundary conditions. 
Special efforts have been put in the computation of the non-linear coefficients derived from the projection onto the normal modes basis.

The temporal part has been exhaustively studied, precising the results of Tobias et al. by systematically considering the effect of damping. Exhaustive analysis of the effect of all the coefficients has been done, as well as the study of uncommon cases which are usually not found in the literature. An unusual jump phenomenon has hence been predicted. Finally, the solution for the deflection has been written, showing that travelling waves are generic when coupled-mode solutions arise. All these results generalize the case considered by Chang, Bajaj and Krousgrill who worked on one-to-one internal resonance for rectangular plates for which $C_{1}=C_{2}$ with particular attention paid to the bifurcation sets and the onset of chaotic behaviour.

The second part of the present paper will be concerned with experimental validations. Experimental resonance curves will be compared to theoretical ones. The case of a residual forcing on configuration (2) will be depicted. This will lead to the occurrence and the observation of a new solution branch which was not studied here. Finally, the approximations considered in the above-presented theory will be systematically discussed.

\section{ACKNOWLEDGMENTS}

The authors would like to thank the developers of the code dstool from the Center for Applied Mathematics at Cornell University. This software has been of great help in the study of the dynamics. The first author would like to thank Paul Manneville for showing him the wild nature encountered in dynamical system theory, as well as Pierre Lanchantin for his last-minute vigilance.

\section{REFERENCES}

1. A. H. NaYfeh and D. T. Mook 1979 Nonlinear Oscillations. New York: John Wiley and Sons.

2. L. Meirovitch 1967 Analytical Methods in Vibrations. New York: MacMillan Publishing Co.

3. S. A. Tobias and R. N. ARNold 1957 Proceedings of the Institution of Mechanical Engineers 171, 669-690. The influence of dynamical imperfection on the vibration of rotating disks.

4. G. J. Efstathiades 1971 Journal of Sound and Vibration 16, 231-253. A new approach to the large-deflection vibrations of imperfect circular disks using Galerkin's procedure.

5. N. YAMAKi 1961 Zeitschrift für Angewandte Mathematik und Mechanik 41, 501-510. Influence of large amplitudes on flexural vibrations of elastic plates.

6. G. C. Kung and Y. H. Pao 1972 Journal of Applied Mechanics 39, 1050-1054. Nonlinear flexural vibration of a clamped circular plate.

7. S. Sridhar, D. T. Mook and A. H. Nayfeh 1975 Journal of Sound and Vibration 41, 359-373. Nonlinear resonances in the forced responses of plates. Part I: symmetric responses of circular plates.

8. J. Hadian and A. H. NaYfeh 1990 Journal of Sound and Vibration 142, 279-292. Modal interaction in circular plates.

9. M. Sathyamoorthy 1996 Applied Mechanical Review Part 2 49, S55-S62. Nonlinear vibrations of plates: an update of recent research.

10. S. A. ToBias 1957 Proceedings of the Institution of Mechanical Engineers 171, 691-701. Free undamped non-linear vibrations of imperfect circular disks.

11. C. J. H. Williams and S. A. Tobias 1963 Journal of Mechanical Engineering Science 5, 325-335. Forced undamped non-linear vibrations of imperfect circular discs.

12. A. Raman and C. D. Mote JR 2001 International Journal of Non-linear Mechanics 36, 261-289. Effects of imperfection on the non-linear oscillations of circular plates spinning near critical speed. 
13. A. Raman and C. D. Mote $\mathbf{J}_{\mathrm{R}} 2001$ International Journal of Non-linear Mechanics 36, 291-305. Experimental studies on the non-linear oscillations of imperfect circular disks spinning near critical speed.

14. D. S. Dugdale 1979 International Journal of Engineering Science 17, 745-756. Non-linear vibration of a centrally clamped rotating disc.

15. S. Sridhar, D. T. Mook and A. H. NaYfeh 1978 Journal of Sound and Vibration 59, 159-170. Nonlinear resonances in the forced responses of plates. Part II: asymmetric responses of circular plates.

16. U. S. Gupta and A. H. Ansari 1998 Journal of Sound and Vibration 215, 231-250. Asymmetric vibrations and elastic stability of polar orthotropic circular plates of linearly varying profile.

17. U. S. Gupta and N. Goyal 1999 Journal of Sound and Vibration 220, 641-657. Forced asymmetric response of linearly tapered circular plates.

18. P. Ribeiro and M. Petyt 2000 International Journal of Non-linear Mechanics 35, 263-278. Nonlinear free vibration of isotropic plates with internal resonance.

19. P. Ribeiro and M. Petyt 1999 Journal of Sound and Vibration 226, 955-983, 985-1010. Geometrical non-linear, steady-state, forced, periodic vibration of plates, part I and II.

20. G. Herrmann 1955 NACA Technical Report 3578. Influence of large amplitudes on flexural vibrations of elastic plates.

21. O. Millet, A. Hhamdouni and A. Ccimetiére 2001 International Journal of Non-linear Mechanics 35. A classification of thin plate models by asymptotic expansion of non-linear threedimensional equilibrium equations.

22. O. Thomas, C. Touzé and A. Chaigne 2000 Proceedings of the 7th International Congress on Sound and Vibration, Garmisch-Partenkirchen. Non-linear resonances in large-deflection vibrations of free-edge circular plates.

23. O. Thomas, C. Touzé and A. Chaigne 2001 Actes du Congrès Francais de Mécanique, Nancy. Sur la vibration à un mode asymétrique des plaques circulaires en grands déplacements.

24. M. GÉRADIN and D. RiXen 1996 Théorie des Vibrations. Paris: Masson; second edition.

25. G. A. Holzapfel 2000 Nonlinear Solid Mechanics. New York: John Wiley and Sons.

26. C. Y. Chin 1980 Nonlinear Analysis of Plates. New York: McGraw-Hill.

27. S. W. Shaw and C. Pierre 1994 Journal of Sound and Vibration 169, 319-347. Normal modes of vibration for non-linear continuous systems.

28. A. H. Nayfeh and S. A. NAyfeh 1994 Transactions of the American Society of Mechanical Engineers Journal of Vibration and Acoustics 116, 129-136. On nonlinear modes of continuous systems.

29. R. Benamar, M. M. K. Bennouna and R. G. White 1991 Journal of Sound and Vibration 164, 295-316. The effects of large vibration amplitudes on the mode shapes and natural frequencies of thin elastic structures. Part II: fully clamped rectangular isotropic plates.

30. T. A. NAYFEH and A. F. VAKakis 1994 International Journal of Non-linear Mechanics 29, 233245. Subharmonic travelling waves in a geometrically non-linear circular plate.

31. K. YASUdA and T. AsAno 1986 Bulletin of JSME 29, 3090-3095. Nonlinear forced oscillations of a rectangular membrane with degenerated modes.

32. S. I. Chang, A. K. Bajaj and C. M. Krousgrill 1993 Nonlinear Dynamics 4, 433-460. Non-linear vibrations and chaos in harmonically excited rectangular plates with one-to-one internal resonance.

33. R. LeWANDOWSKI 1996 Meccanica 31, 323-346. On beams membranes and plates vibration backbone curves in cases of internal resonance.

34. X. L. YANG and P. R. Sethna 1991 International Journal of Non-linear Mechanics 26, 199-220. Local and global bifurcations in parametrically excited vibrations of nearly square plates.

35. A. H. Nayfeh and B. Balachandran 1989 Applied Mechanical Review Part 2 42, S175-S201. Modal interactions in dynamical and structural systems.

36. C. Touzé 2000 Ph.D. Thesis, Université Pierre et Marie Curie, Paris VI. Analyse et modélisation de signaux vibratoires et acoustiques chaotiques. Application aux intruments de percussion nonlinéaires.

37. C. Touzé and A. Chaigne 2000 Acta Acustica 86, 557-567. Lyapunov exponents from experimental time series: application to cymbal vibrations.

38. J. Guckenheimer, M. R. Myers, F. J. Wicklin and P. A. Worfolk 1995 Technical Report, Center for Applied Mathematics, Cornell University, Ithaca, NY 14853, U.S.A. DsTool: a dynamical system toolkit with an interactive graphical interface. 
39. M. Amabili, F. Pellicano and A. F. Vakakis 2000 Journal of Vibration and Acoustics $\mathbf{1 2 2}$, 346-354. Nonlinear vibrations and multiple resonances of fluid-filled circular shells. Part 1: equations of motion and numerical results.

40. P. Manneville 1990 Dissipative Structures and Weak Turbulence. New York: Academic Press.

41. A. Leissa 1993 Vibration of Plates. New York: Acoustical Society of America.

\section{APPENDIX A: EXPRESSION OF THE MODE SHAPES}

\section{A.1. FREE EDGE (MODES $\left.\Phi_{\alpha}\right)$}

Following from the linear part of equation (12a), and boundary conditions (9b, c), the spatial part of the solution $w$ satisfies, for all $\theta$ and $t$,

$$
\begin{gathered}
\left(\Delta \Delta-\zeta^{4}\right) \Phi=0, \\
\Phi_{, r r}+v \Phi_{, r}+v \Phi_{, \theta \theta}=0 \quad \text { at } r=1, \\
\Phi_{, r r r}+\Phi_{, r r}-\Phi_{, r}+(2-v) \Phi_{, r \theta \theta}-(3-v) \Phi_{, \theta \theta}=0 \quad \text { at } r=1, \\
\Phi(r=0) \text { is bounded }
\end{gathered}
$$

with $\zeta^{4}=\omega^{2}$. The solutions of the previous set are separated in $r$ and $\theta$, and are written as

$$
\begin{gathered}
\Phi_{0 n}(r, \theta)=R_{0 n}(r) \quad \text { for } k=0, \\
\Phi_{k n 1}(r, \theta) \\
\Phi_{k n 2}(r, \theta)
\end{gathered}\left|=R_{k n}(r)\right| \begin{array}{ll}
\cos k \theta & \text { for } k>0 \\
\sin k \theta &
\end{array}
$$

with

$$
R_{k n}(r)=\kappa_{k n}\left[\mathbf{J}_{k}\left(\zeta_{k n} r\right)-\frac{\tilde{\mathbf{J}}_{k}\left(\zeta_{k n}\right)}{\tilde{\mathbf{I}}_{k}\left(\zeta_{k n}\right)} \mathbf{I}_{k}\left(\zeta_{k n} r\right)\right],
$$

where $\mathbf{J}_{k}$ is the Bessel functions of order $k$ of the first kind, $\mathrm{I}_{k}(x)=\mathbf{J}_{k}(\mathrm{i} x)$ with $\mathrm{i}=\sqrt{-1}$, and $\tilde{\mathbf{J}}_{k}$ and $\tilde{\mathbf{I}}_{k}$ are defined as follows:

$$
\begin{aligned}
& \tilde{\mathbf{J}}_{k}(x)=x^{2} \mathbf{J}_{k-2}(x)+x(v-2 k+1) \mathbf{J}_{k-1}(x)+k(k+1)(1-v) \mathbf{J}_{k}(x), \\
& \tilde{\mathbf{I}}_{k}(x)=x^{2} \mathbf{I}_{k-2}(x)+x(v-2 k+1) \mathbf{I}_{k-1}(x)+k(k+1)(1-v) \mathbf{I}_{k}(x) .
\end{aligned}
$$

$\kappa_{k n}$, a constant, is chosen so that

$$
\iint_{(\mathscr{S})} \Phi_{k n}^{2} \mathrm{~d} S=1 .
$$

$\zeta_{k n}$ is the $\tilde{n}$ th solution of the following equation:

$$
\begin{aligned}
& \tilde{\mathbf{I}}_{k}(\zeta)\left[\zeta^{3} \mathbf{J}_{k-3}(\zeta)+\zeta^{2}(4-3 k) \mathbf{J}_{k-2}(\zeta)\right. \\
& \left.+\zeta k(k(1+v)-2) \mathbf{J}_{k-1}(\zeta)+k^{2}(1-v)(1+k) \mathbf{J}_{k}(\zeta)\right] \\
& -\tilde{\mathbf{J}}_{k}(\zeta)\left[\zeta^{3} \mathbf{I}_{k-3}(\zeta)+\zeta^{2}(4-3 k) \mathbf{I}_{k-2}(\zeta)\right. \\
& \left.+\zeta k(k(1+v)-2) \mathbf{I}_{k-1}(\zeta)+k^{2}(1-v)(1+k) \mathbf{I}_{k}(\zeta)\right]=0 .
\end{aligned}
$$

$k$ is found to be the number of nodal radii. Because of the free-edge boundary conditions, the edge of the plate is not a nodal circle, and mode $\Phi_{10}$ is a rigid-body mode. So, the number $n$ of nodal circles is not equal to $\tilde{n}$. In fact, for $k=1, n=\tilde{n}$ and for $k \neq 1, n=\tilde{n}-1$ [41]. Computed values of the $\zeta_{k n}$ can be found in reference [15] and their squared values $\left(\omega_{k n}=\zeta_{k n}^{2}\right)$ are given in Table 1 . 


\section{A.2. CLAMPED EDGE (MODES $\Psi_{s}$ )}

Combining equation (18) and boundary conditions (19), the spatial part of $F$ satisfies, for all $\theta$ and $t$,

$$
\begin{gathered}
\left(\Delta \Delta-\xi^{4}\right) \Psi=0, \quad \Psi=0, \quad \text { at } r=1, \\
\Psi_{, r}=0, \quad \text { at } r=1, \quad \Psi(r=0) \text { is finite. }
\end{gathered}
$$

The solutions of the previous set are separated in $r$ and $\theta$ :

$$
\begin{gathered}
\Psi_{0 m}(r, \theta)=S_{0 m}(r) \quad \text { for } l=0, \\
\Psi_{l m 1}(r, \theta) \\
\Psi_{l m 2}(r, \theta)
\end{gathered}\left|=S_{l m}(r)\right| \begin{array}{ll}
\cos l \theta & \text { for } l>0 \\
\sin l \theta &
\end{array}
$$

with

$$
S_{l m}(r)=\lambda_{l m}\left[\mathbf{J}_{l}\left(\xi_{l m} r\right)-\frac{\mathbf{J}_{l}\left(\xi_{l m}\right)}{\mathbf{I}_{l}\left(\xi_{l m}\right)} \mathbf{I}_{l}\left(\xi_{l m} r\right)\right],
$$

where the $\xi_{l m}$ is the $m$ th solution of the following equation:

$$
\mathbf{J}_{l-1}(\xi) \mathbf{I}_{l}(\xi)-\mathbf{I}_{l-1}(\xi) \mathbf{J}_{l}(\xi)=0 .
$$

Computed values of the $\xi_{l m}$ can be found in reference [41]. $\lambda_{l m}$, a constant, is chosen so that

$$
\iint_{(\mathscr{S})} \Psi_{l m}^{2} \mathrm{~d} S=1
$$

$l$ and $m$ correspond to the numbers of nodal radii and circles, respectively.

We can remark that if $k \geqslant 1$ (or $l \geqslant 1$ ), each $\zeta_{k n}$ (or $\xi_{l m}$ ) is associated to the two degenerated modes of equation (A.2) (or equation (A.8)), one in cosine, the other in sine [2].

\section{APPENDIX B: CALCULATION OF COEFFICIENTS $\Gamma$}

\section{B.1. THE CASE OF AN AXISYMMETRIC MODE $\Phi$}

In this section, all transverse modes $\Phi$ found in equation (25) equal $\Phi_{0 n}$. Taking equations (A.5) and (A.11), and definition (2) of the functional $L$ into account, the only coefficient $\Gamma$ obtained from equation (23) is

$$
\Gamma=-\frac{1}{2} \sum_{l, m} \xi_{l m}^{-4}\left[\mathscr{I}_{1}(\operatorname{lm}) \mathscr{J}_{1}(\operatorname{lm})+\mathscr{I}_{2}(\operatorname{lm}) \mathscr{J}_{2}(\operatorname{lm})\right]
$$

with

$$
\begin{aligned}
& \begin{array}{l}
\mathscr{I}_{1}(\operatorname{lm}) \\
\mathscr{I}_{2}(\operatorname{lm})
\end{array}\left|=\iint_{(\mathscr{S})} L\left(\Phi_{0 n}, \Phi_{0 n}\right)\right| \begin{array}{l}
\Psi_{l m 1} \\
\Psi_{l m 2}
\end{array} \mid \mathrm{d} S \\
& =\int_{0}^{1} L\left(R_{0 n}, R_{0 n}\right) S_{l m} r \mathrm{~d} r \int_{0}^{2 \pi}\left|\begin{array}{c}
\cos l \theta \\
\sin l \theta
\end{array}\right| \mathrm{d} \theta, \\
& \left.\begin{array}{l}
\mathscr{J}_{1}(\operatorname{lm}) \\
\mathscr{J}_{2}(\operatorname{lm})
\end{array}\left|=\iint_{(\mathscr{S})} \Phi_{0 n} L \quad \Phi_{0 n},\right| \begin{array}{l}
\Psi_{l m 1} \\
\Psi_{l m 2}
\end{array} \mid\right) \mathrm{d} S \\
& =\int_{0}^{1} R_{0 n} L\left(R_{0 n}, S_{l m}\right) r \mathrm{~d} r \int_{0}^{2 \pi}\left|\begin{array}{c}
\cos l \theta \\
\sin l \theta
\end{array}\right| \mathrm{d} \theta .
\end{aligned}
$$


Therefore, because of the value of the integrals of sine and cosine from 0 to $2 \pi$ in the above equations, the only non-zero $\mathscr{I}_{\alpha}$ and $\mathscr{J}_{\alpha}$ are

$$
\begin{gathered}
\mathscr{I}_{1}(0 m)=4 \pi \int_{0}^{1} R_{0 n}^{\prime \prime}(r) R_{0 n}^{\prime}(r) S_{0 m}(r) \mathrm{d} r \\
\mathscr{J}_{1}(0 m)=2 \pi \int_{0}^{1} R_{0 n}(r)\left[R_{0 n}^{\prime \prime}(r) S_{0 m}^{\prime}(r)+R_{0 n}^{\prime}(r) S_{0 m}^{\prime \prime}\right] \mathrm{d} r
\end{gathered}
$$

where $R^{\prime}(r)$ denotes the derivative with respect to $r$ of function $R$ of $r$. As a consequence, one can note that the only longitudinal $(\Psi)$ modes involved are the axisymmetric ones. This result confirms the hypothesis of axisymmetric vibration used in reference [7].

As a conclusion, the coefficient $\Gamma$ can be calculated using the following sum over all axisymmetric modes $\Psi_{0 m}$ :

$$
\Gamma=-\frac{1}{2} \sum_{m=1}^{+\infty} \xi_{0 m}^{-4} \mathscr{I}_{1}(0 m) \mathscr{J}_{1}(0 m)
$$

where the values of $\mathscr{I}_{1}$ and $\mathscr{J}_{1}$ are given by equations (B.3a, b). Computed values of $\Gamma$ for the first three modes $\Phi_{0 n}$ can be found in Table 1 .

\section{B.2. THE CASE OFAN ASYMMETRIC MODE $\Phi$}

In this section, the transverse modes $\Phi$ of equation (25) can equal either $\Phi_{1}=\Phi_{k n 1}$ or $\Phi_{2}=\Phi_{k n 2}$, which are the two preferential modes. We then need to determine the following 16 coefficients:

$$
\Gamma_{\alpha \beta \tilde{\alpha} \tilde{\beta}}=-\frac{1}{2} \sum_{l, m, \gamma} \xi_{l m}^{-4} \mathscr{I}_{\alpha \beta \gamma}(\operatorname{lm}) \mathscr{J}_{\tilde{\alpha} \tilde{\beta} \gamma}(\operatorname{lm}), \quad \alpha, \beta, \tilde{\alpha}, \tilde{\beta}, \gamma \in\{1,2\}^{5},
$$

where (A.5) and (A.11) have been used and

$$
\begin{aligned}
& \mathscr{I}_{\alpha \beta \gamma}(\operatorname{lm})=\iint_{(\mathscr{S})} L\left(\Phi_{k n \alpha}, \Phi_{k n \beta}\right) \Psi_{l m \gamma} \mathrm{d} S=-2 \mathrm{I}_{1}(k n, \operatorname{lm}) \Pi_{\alpha \beta \gamma}^{(1)}+2 \mathrm{I}_{2}(k n, l m) \Pi_{\alpha \beta \gamma}^{(2)}, \\
& \mathscr{J}_{\tilde{\alpha} \tilde{\beta} \gamma}(\operatorname{lm})=\iint_{(\mathscr{S})} \Phi_{k n \tilde{\alpha}} L\left(\Phi_{k n \tilde{\beta}}, \Psi_{l m \gamma}\right) \mathrm{d} S=-2 \mathrm{~J}_{1}(k n, l m) \Pi_{\tilde{\alpha} \tilde{\beta} \gamma}^{(3)}+\mathbf{J}_{2}(k n, l m) \Pi_{\tilde{\alpha} \tilde{\beta} \gamma}^{(2)}
\end{aligned}
$$

with

$$
\begin{gathered}
\mathrm{I}_{1}(k n, l m)=\int_{0}^{1}\left[\frac{R_{k n}^{\prime 2}}{r^{2}}-2 \frac{R_{k n} R_{k n}^{\prime}}{r^{3}}+\frac{R_{k n}^{2}}{r^{4}}\right] S_{l m} \mathrm{~d} r \\
\mathrm{I}_{2}(k n, l m)=\int_{0}^{1} R_{k n}^{\prime \prime}\left[R_{k n}^{\prime}-k^{2} \frac{R_{k n}}{r}\right] S_{l m} \mathrm{~d} r \\
\mathrm{~J}_{1}(k n, l m)=\int_{0}^{1} k l R_{k n}\left[\frac{R_{k n}^{\prime} S_{l m}^{\prime}}{r}+\frac{R_{k n}^{\prime} S_{l m}+R_{k n} S_{l m}^{\prime}}{r^{2}}+\frac{R_{k n} S_{l m}}{r^{3}}\right] \mathrm{d} r \\
\mathrm{~J}_{2}(k n, l m)=\int_{0}^{1} R_{k n}\left[R_{k n}^{\prime \prime}\left(S_{l m}^{\prime}-l^{2} \frac{S_{l m}}{r}\right)+S_{l m}^{\prime \prime}\left(R_{k n}^{\prime}-k^{2} \frac{R_{k n}}{r}\right)\right] \mathrm{d} r .
\end{gathered}
$$


The $R_{k n}(r)$ and $S_{l m}(r)$ in the above equations are defined by equations (A.3) and (A.9). The factors $\Pi_{\alpha \beta \gamma}^{\mathrm{i})}(k, l)$ are the following integrals:

$$
\begin{gathered}
\Pi_{\alpha \beta \gamma}^{(1)}(k, l)=\int_{0}^{2 \pi}\left|\begin{array}{c}
-\sin k \theta \\
\cos k \theta
\end{array}\right| \begin{array}{c}
-\sin k \theta \\
\cos k \theta
\end{array}|| \begin{array}{c}
\cos l \theta \\
\sin l \theta
\end{array} \mid \mathrm{d} \theta, \\
\Pi_{\alpha \beta \gamma}^{(2)}(k, l)=\int_{0}^{2 \pi}\left|\begin{array}{c}
\cos k \theta \\
\sin k \theta
\end{array}\right| \begin{array}{c}
\cos k \theta \\
\sin k \theta
\end{array}|| \begin{array}{c}
\cos l \theta \\
\sin l \theta
\end{array} \mid \mathrm{d} \theta, \\
\Pi_{\alpha \beta \gamma}^{(3)}(k, l)=\int_{0}^{2 \pi}\left|\begin{array}{c}
\cos k \theta \\
\sin k \theta
\end{array}\right|\left|\begin{array}{c}
-\sin k \theta \\
\cos k \theta
\end{array}\right|\left|\begin{array}{c}
-\sin l \theta \\
\cos k \theta
\end{array}\right| \mathrm{d} \theta .
\end{gathered}
$$

The above notations mean that all $\Pi_{\alpha \beta \gamma \gamma}$ are obtained by making the product of three functions sine and/or cosine, each one being taken in a column of the above matrices, the values of $\alpha, \beta$ and $\gamma$ determining the line $(\alpha=2$ corresponds to the term 21 , of the second line of the first column, $\gamma=1$ to the term $13 \ldots)$.

One can show that the only non-zero values of the $\Pi_{\alpha \beta \gamma}$ are obtained for $l=2 k$ or 0 , which means that only the $\Psi_{0 m}$ and $\Psi_{(2 k) m}$ are involved in the sum of equation (B.5).

Table B.1 summarizes the values of the factors $\mathscr{I}_{\alpha \beta \gamma}$ and $\mathscr{J}_{\alpha \beta \gamma}$ as a function, on the one hand of $\alpha, \beta, \gamma$ and on the other hand of the value of $l$ compared to $k$. First, one can see that the products $\mathscr{I}_{\alpha \alpha \gamma} \mathscr{J}_{\alpha \beta \gamma}$ and $\mathscr{I}_{\alpha \beta \gamma} \mathscr{J}_{\alpha \alpha \gamma}$ with $\alpha \neq \beta$ and for all $l$ are always zero. This implies that

$$
\Gamma_{1112}=\Gamma_{1121}=\Gamma_{2212}=\Gamma_{2221}=\Gamma_{1211}=\Gamma_{2111}=\Gamma_{1222}=\Gamma_{2122}=0 .
$$

Moreover, the non-zero $\Gamma$ follows

$$
\begin{gathered}
\Gamma_{1111}=\Gamma_{2222}=\Gamma_{0}+\Gamma^{\star}, \quad \Gamma_{1122}=\Gamma_{2211}=\Gamma_{0}-\Gamma^{\star}, \\
\Gamma_{1212}=\Gamma_{2121}=\Gamma_{1221}=\Gamma_{2112}=\Gamma^{\star}
\end{gathered}
$$

\begin{tabular}{|c|c|c|c|c|}
\hline & \multicolumn{2}{|c|}{ Mode $\Psi_{l m 1}$ in cosine $(\gamma=1)$} & \multicolumn{2}{|c|}{ Mode $\Psi_{\operatorname{lm} 2}$ in sine $(\gamma=2)$} \\
\hline \multirow[t]{3}{*}{$\alpha=\beta$} & $l \notin\{0,2 k\}$ & $\begin{array}{l}\mathscr{I}_{111}=\mathscr{I}_{221}=0 \\
\mathscr{J}_{111}=\mathscr{J}_{221}=0\end{array}$ & $\forall l$ & $\begin{array}{l}\mathscr{I}_{112}=\mathscr{I}_{222}=0 \\
\mathscr{J}_{112}=\mathscr{J}_{222}=0\end{array}$ \\
\hline & $l=0$ & $\begin{array}{l}\mathscr{I}_{111}=\mathscr{I}_{221}=-2 \pi k^{2} \mathrm{I}_{1}+2 \pi \mathrm{I}_{2} \\
\mathscr{J}_{111}=\mathscr{J}_{221}=\pi \mathrm{J}_{2}\end{array}$ & & \\
\hline & $l=2 k$ & $\begin{aligned} \mathscr{I}_{111} & =-\mathscr{I}_{221}=\pi k^{2} \mathrm{I}_{1}+\pi \mathrm{I}_{2} \pi \\
\mathscr{J}_{111} & =-\mathscr{J}_{221}=-2 \pi k^{2} \mathrm{~J}_{1}+\frac{-}{2} \mathbf{J}_{2}\end{aligned}$ & & \\
\hline \multirow[t]{2}{*}{$\alpha \neq \beta$} & $\forall l$ & $\begin{array}{l}\mathscr{I}_{121}=\mathscr{I}_{211}=0 \\
\mathscr{J}_{121}=\mathscr{J}_{211}=0\end{array}$ & $l \neq 2 k$ & $\begin{array}{l}\mathscr{I}_{122}=\mathscr{I}_{122}=0 \\
\mathscr{J}_{122}=\mathscr{J}_{212}=0\end{array}$ \\
\hline & & & $l=2 k$ & $\begin{array}{l}\mathscr{I}_{122}=\mathscr{I}_{212}=\pi k^{2} \mathrm{I}_{1}+\pi \mathrm{I}_{2} \\
\mathscr{J}_{122}=\mathscr{I}_{122}=-2 \pi k^{2} \mathrm{~J}_{1}+\frac{\pi}{2} \mathrm{~J}_{2}\end{array}$ \\
\hline
\end{tabular}

with

$$
\Gamma_{0}=-\pi^{2} \sum_{m=1}^{+\infty} \xi_{0 m}^{-4}\left(\mathbf{I}_{20}-k^{2} \mathbf{I}_{10}\right) \mathbf{J}_{20}
$$

TABLE B. 1

Coefficients $\mathscr{I}_{\alpha \beta \gamma}$ and $\mathscr{J}_{\alpha \beta \gamma}$ 


$$
\begin{gathered}
\left.\Gamma^{\star}=-\frac{\pi^{2}}{2} \sum_{m=1}^{+\infty} \xi_{(2 k) m}^{-4}\left(k^{2} \mathbf{I}_{1}^{\star}+\mathrm{I}_{2}^{\star}\right) \frac{\mathbf{J}_{2}^{\star}}{2}-2 k^{2} \mathbf{J}_{1}^{\star}\right), \\
\mathrm{I}_{i 0}=\mathrm{I}_{i}(k n, 0 m), \quad \mathbf{J}_{i 0}=\mathbf{J}_{i}(k n, 0 m), \\
\mathrm{I}_{i}^{\star}=\mathbf{I}_{i}(k n,(2 k) m), \quad \mathbf{J}_{i}^{\star}=\mathbf{J}_{i}(k n,(2 k) m) .
\end{gathered}
$$

$\Gamma_{0}\left(\Gamma^{\star}\right)$ results from the sum over the axisymmetric longitudinal modes $\Psi_{0 m}$ (the asymmetric longitudinal modes with $l=2 k$ nodal radii).

To obtain equations $(27 \mathrm{~b}, \mathrm{c})$, one has first to determine the non-linear terms resulting from set (23). They are listed below:

$$
\begin{aligned}
& \text { equation in } q_{1}: \Gamma_{11} q_{1}^{3}+\Gamma_{12} q_{2}^{3}+C_{11} q_{1} q_{2}^{2}+C_{12} q_{1}^{2} q_{2}, \\
& \text { equation in } q_{2}: \quad \Gamma_{21} q_{1}^{3}+\Gamma_{22} q_{2}^{3}+C_{21} q_{1} q_{2}^{2}+C_{22} q_{1}^{2} q_{2},
\end{aligned}
$$

where

$$
\begin{aligned}
& \Gamma_{11}=\Gamma_{1111}, \quad \Gamma_{22}=\Gamma_{2222}, \quad \Gamma_{12}=\Gamma_{2212}, \quad \Gamma_{21}=\Gamma_{1121}, \\
& C_{11}=\Gamma_{1212}+\Gamma_{2112}+\Gamma_{2211}, \quad C_{22}=\Gamma_{2121}+\Gamma_{1221}+\Gamma_{1122}, \\
& C_{12}=\Gamma_{1211}+\Gamma_{2111}+\Gamma_{1112}, \quad C_{21}=\Gamma_{1222}+\Gamma_{2122}+\Gamma_{2221} .
\end{aligned}
$$

Then, with equations (B.10-B.12), one finally obtains

$$
\begin{gathered}
\Gamma_{12}=\Gamma_{21}=C_{12}=C_{21}=0, \\
\Gamma_{11}=\Gamma_{22}=C_{11}=C_{22}=\Gamma_{0}+\Gamma^{\star}=\Gamma .
\end{gathered}
$$

\section{APPENDIX C: NOMENCLATURE}

$a$
$h$
$\rho$
$v$
$E$
$p(r, \theta, t)$
$w(r, \theta, t)$
$F$
$N_{\alpha \beta}$
$\pi_{\alpha \beta}$
$M_{r}, M_{r} \theta$
$Q_{r}$
$\Phi_{p}$
$\Psi_{p}$
$\mu_{p}$
$\omega_{p}$
$\sigma_{1}$
$\sigma_{2}$

radius of the plate thickness of the plate density of the plate the Poisson ratio Young's modulus external load applied to the plate vertical displacement

force function membrane force per unit length, $(\alpha, \beta) \in r, \theta$ components of the second Piola-Kirchhoff stress tensor bending and twisting moments transverse shear force $p$ th linear natural mode for the plate with free edge $p$ th linear natural mode for the plate with clamped edge dimensionless modal damping of the $p$ th mode dimensionless angular frequency of the $p$ th mode internal detuning between the two preferential configurations detuning between the excitation pulsation and the pulsation of the first configuration 